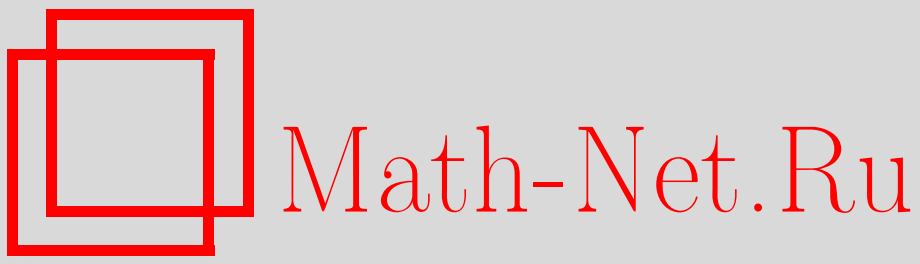

Ч.-Х. Ли, Косые сверточные полугруппы и связанные с ними процессы с иммиграцией, Теория вероятн. и ее примен., 2001, том 46, выпуск 2, 247-274 DOI: https://doi.org/10.4213/tvp3917

Использование Общероссийского математического портала MathNet.Ru подразумевает, что вы прочитали и согласны с пользовательским соглашением

http://www.mathnet.ru/rus/agreement

Параметры загрузки:

IP : 54.237 .206 .68

26 апреля 2023 г., $17: 05: 17$

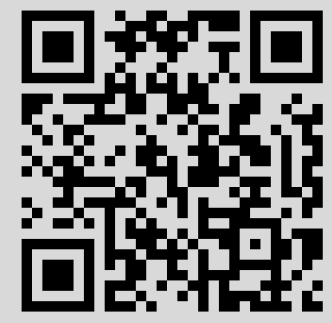




\title{
КОСЫЕ СВЕРТОЧНЫЕ ПОЛУГРУППЫ И СВЯЗАННЫЕ С НИМИ ПРОЦЕССЫ С ИММИГРАЦИЕЙ
}

\begin{abstract}
С помощью косых сверточных полугрупп описывается особый тип иммиграции, связанной с мерозначными ветвящимися процессами. Приводится характеризация общей неоднородной косой сверточной полугруппы в терминах входных вероятностных законов. Соответствующий процесс с иммиграцией строится путем суммирования мерозначных траекторий процесса Кузнецова, определенного правилом вхождения. Затем изучается поведение процесса Кузнецова, что дает представление о структуре траекторий процесса̀ с иммиграцией. Некоторые известные результаты об избыточных мерах формулируются в терминах стационарных процессов с иммиграцией.
\end{abstract}

Ключевые слова и фразы: мерозначный ветвяшийся процесс, суперпроцесс, процесс с иммиграцией, косая сверточная полугруппа, входной закон, правило вхождения, избыточная мера, мера Кузнецова.

1. Введение. Пусть $E-$ лузинское топологическое пространство (т.е. гомеоморфизм борелевского подмножества компактного метрического пространства) с борелевской $\sigma$-алгеброй $\mathscr{B}(E)$. Пусть $B(E)$ обозначает множество ограниченных $\mathscr{B}(E)$-измеримых функций на $E$, а $B(E)^{+}$- подпространство $B(E)$, содержащее неотрицательные функции. Символом $M(E)$ мы обозначим пространство конечных мер на $(E, \mathscr{B}(E))$ с топологией слабой сходимости. Для $f \in B(E)$ и $\mu \in M(E)$ мы будем обозначать $\mu(f)$ интеграл $\int_{E} f d \mu$. Предположим, что $\left(P_{t}\right)_{t \geqslant 0}-$ переходная полугруппа правого борелевского процесса $\xi$ с пространством состояний $E$, а $\phi(\cdot, \cdot)$ - механизм ветвления, задаваемый соотношением

$$
\phi(x, z)=b(x) z+c(x) z^{2}+\int_{0}^{\infty}\left(e^{-z u}-1+z u\right) m(x, d u),
$$

Zong- $H u$ Li. Skew convolution semi-groups and related immigration processes.

(c) Перевод на русский язык, Научное издательство «ТВП», 2001.

${ }^{*}$ Department of Mathematics, Beijing Normal University, Beijing 100875, China; e-mail: lizh@math.carleton.ca

1) Работа выполнена при поддержке National Natural Science Foundation of China (№ 19361060). 
$x \in E, z \geqslant 0$, где $b \in B(E), c \in B(E)^{+}$и $\left(u \wedge u^{2}\right) m(x, d u)-$ ограниченное ядро, действующее из $E$ в $(0, \infty)$. Тогда для любого $f \in B(E)^{+}$ эволюционное уравнение

$$
V_{t} f(x)+\int_{0}^{t} d s \int_{E} \phi\left(y, V_{s} f(y)\right) P_{t-s}(x, d y)=P_{t} f(x)
$$

$t \geqslant 0, x \in E$, имеет единственное решение $V_{t} f \in B(E)^{+}$, и существует марковская полугруппа $\left(Q_{t}\right)_{t \geqslant 0}$ на $M(E)$ такая, что

$$
\int_{M(E)} e^{-\nu(f)} Q_{t}(\mu, d \nu)=\exp \left\{-\mu\left(V_{t} f\right)\right\}
$$

для всех $t \geqslant 0, \mu \in M(E)$ и $f \in B(E)^{+}$. Марковский процесс $X$ с переходной полугруппой $\left(Q_{t}\right)_{t \geqslant 0}$ называется суперпрочессом Доусона-Ватанабе с параметрами $(\xi, \phi)$. При наших предположениях процесс $X$ имеет правую борелевскую реализацию; см. [11] и $[12] .(\xi, \phi)$-суперпроцесс является математической моделью эволюции популяции в некоторой области, см., например, [3] и [4]. При изучении ситуации, в которой имеются дополнительные источники, из которых происходит иммиграция индивидуумов в рассматриваемую область, необходимо ввести ветвящиеся процессы с иммиграцией. Такой тип модифицированных процессов хорошо известен по литературе о ветвящихся процессах, см., например, [1], [5], $[8],[21],[24]$ и [32].

В [25] класс мерозначных процессов с иммиграцией описан следующим образом. Пусть $\left(N_{t}\right)_{t \geqslant 0}$ - семейство вероятностных мер на $M(E)$. Назовем $\left(N_{t}\right)_{t \geqslant 0}$ косой сверточной полугруппой, ассоциированной с $X$ или $\left(Q_{t}\right)_{t \geqslant 0}$, если

$$
N_{r+t}=\left(N_{r} Q_{t}\right) * N_{t}, \quad r, t \geqslant 0,
$$

где «*» обозначает операцию свертки. Соотношение (1.4) имеет место тогда и только тогда, когда

$$
Q_{t}^{N}(\mu, \cdot):=Q_{t}(\mu, \cdot) * N_{t}, \quad t \geqslant 0, \mu \in M(E),
$$

определяет марковскую полугруппу $\left(Q_{t}^{N}\right)_{t \geqslant 0}$ на $M(E)$. Марковский процесс называется прочессом с иммиграчией, ассоциированным с $X$, если он имеет переходную полугруппу $\left(Q_{t}^{N}\right)_{t \geqslant 0}$. Интуитивный смысл процесса с иммиграцией ясен из (1.5), а именно, $Q_{t}(\mu, \cdot)$ - это распределение потомков тех индивидуумов, распределение которых в нулевой момент времени равно $\mu \in M(E)$, а $N_{t}$ - распределение потомков индивидуумов, иммигрировавших в $E$ за время $(0, t]$. Очевидно, $(1.5)$ дает общее описание иммиграции, независимой от «внутреннего» населения.

Бо́льшая часть теории суперпроцессов Доусона-Ватанабе переносится на связанные с ними процессы с иммиграцией и может быть построена с помощью методов, очень близких к тем, которые использовались в [3] и [4]. Однако интересно, что, как видно из (1.4) и (1.5), 
процессы с иммиграцией обладают многими дополнительными структурами. Заметим, что соотношение (1.5) аналогично построению переходной полугруппы Леви по обычной сверточной полугруппе. Хорошо известно, что сверточная полугруппа на евклидовом пространстве однозначно определяется безгранично делимой вероятностной мерой. Как показано в [25], косую сверточную полугруппу можно охарактеризовать в терминах безгранично делимого входного закона. Таким образом, процессы с иммиграцией могут рассматриваться как обобщенные формы хорошо известных процессов Леви. Другими примерами процессов с иммиграцией являются квадраты бесселевых диффузий и радиальные составляющие диффузий Орнштейна-Уленбека, см. [21] и [33]. Приведенное выше описание включает также новые классы процессов, ранее не рассматривавшиеся, см. [26], [27] и [28].

В моделях иммиграции, изучавшихся ранее, обычно предполагалось, что иммигранты прибывают в $E$ в соответствии со случайной мерой на $\mathbf{R} \times E$. При описании в терминах косой сверточной полугруппы сценарии не совсем ясны. Наличие этого слабого места и стало мотивировкой данной работы. Главная цель данной статьи - дать интерпретацию косых сверточных полугрупп с помощью построения мерозначных процессов с иммиграцией и анализа их траекторий. Вначале мы доказываем, что общая неоднородная косая сверточная полугруппа может быть разложена на три компоненты, включающие, соответственно, счетное семейство входных законов, счетное семейство замкнутых входных законов и континуальное семейство безгранично делимых вероятностных законов с рассеянной мерой на пространстве индексов. Затем мы приводим конструкцию определенных выше процессов с иммиграцией с помошью отбора мерозначных траекторий со случайными моментами рождения и гибели. Наша конструкция основана на наблюдении, что любая косая сверточная полугруппа определяется непрерывной возрастающей мерозначной траекторией $\left(\gamma_{t}\right)_{t \geqslant 0}$ и правилом вхождения $\left(G_{t}\right)_{t \geqslant 0}$. Из этого факта вытекает естественное разложение иммиграции на две части на детерминированную часть, определяемую траекторией $\left(\gamma_{t}\right)_{t \geqslant 0}$, и случайную часть, определяемую правилом $\left(G_{t}\right)_{t \geqslant 0}$. Вторая часть обычно является неоднородным процессом с иммиграцией и может быть построена путем суммирования траекторий $\left\{w_{t}: \alpha<t<\beta\right\}$ в ассоциированном процессе Кузнецова, см. [22]. С помощью анализа асимптотического поведения траекторий $\left\{w_{t}: \alpha<t<\beta\right\}$ в окрестности времени рождения $\alpha=\alpha(w)$ мы показываем, что почти все эти траектории начинают распространяться в расширении $E_{D}^{T}$ исходного пространства, включая те, точки роста которых составляют множество меры нуль в этом пространстве. В сочетании с нашей конструкцией процесса с иммиграцией это дает полное описание изучаемого явления. В некоторых особых случаях безгранично делимый входной закон для $(\xi, \phi)$-суперпроцесса соот- 
ветствует $\sigma$-конечному входному закону, и ассоциированный процесс с иммиграцией может быть построен с помощью однородного траекторнозначного пуассоновского случайного процесса, характеристическая мера которого является марковской мерой, определяемой входным законом. Эта конструкция хорошо зарекомендовала себя при изучении процессов с иммиграцией, см., например, [26], [29] и [32]. В качестве дополнительных применений наших построений мы приводим формулировки некоторых хорошо известных результатов об избыточных мерах в терминах стационарных процессов с иммиграцией.

Статья устроена следующим образом. Пункт 2 содержит предварительные сведения. В п. 3 мы доказываем теорему о разложении для неоднородных косых сверточных полугрупп. Конструкция процессов с иммиграцией с использованием процессов Кузнецова приведена в п. 4. «Почти наверное»-поведение процессов Кузнецова изучается в п. 5. В п. 6 мы обсуждаем стационарные процессы с иммиграцией, определяемые избыточными мерами.

2. Предварительные сведения. Напомним, что $M(E)$ - это пространство конечных борелевских мер на лузинском топологическом пространстве $E$. Хорошо известно, что пространство $M(E)$, снабженное топологией слабой сходимости, также является лузинским пространством. Пусть $M(E)^{\circ}=M(E) \backslash\{0\}$, где 0 обозначает нулевую меру. Для вероятностной меры $F$ на $M(E)$ зададим ее функционал Лапласа соотношением

$$
L_{F}(f):=\int_{M(E)} e^{-\nu(f)} F(d \nu), \quad f \in B(E)^{+},
$$

которое однозначно определяет $F$. Хорошо известно, что $F$ безгранично делима тогда и только тогда, когда

$$
L_{F}(f)=\exp \left\{-\eta(f)-\int_{M(E)^{\circ}}\left(1-e^{-\nu(f)}\right) H(d \nu)\right\},
$$

$f \in B(E)^{+}$, где $\eta \in M(E)$ и $[1 \wedge \nu(E)] H(d \nu)$ является конечной мерой на $M(E)^{\circ}$ (см., например, [20]). Мы пишем $F=I(\eta, H)$, если $F$ определяется соотношением (2.2).

Предположим, что $X=\left(W, \mathscr{G}, \mathscr{G}_{r, t}, X_{t}, \mathbf{Q}_{r, \mu}\right)$ - марковский процесс в $M(E)$ с переходной полугруппой $\left(Q_{r, t}\right)_{r \leqslant t}$. Пусть

$$
V_{r, t} f(x):=-\ln \int_{M(E)} e^{-\nu(f)} Q_{r, t}\left(\delta_{x}, d \nu\right),
$$

$r \leqslant t, x \in E, f \in B(E)^{+}$, где $\delta_{x}$ обозначает единичную массу, сосредоточенную в точке $x \in E$. В данной статье мы всегда считаем, что для любых $r \leqslant t$ и $f \in B(E)^{+}$сужение функции $V_{s, t} f(x)$ от $(s, x)$ на $[r, t] \times E$ ограничено и измеримо. Мы также предполагаем, что $V_{r, t} f(x)$ непрерывна справа по $t \geqslant r$ при $f \in C(E)^{+}$, т.е. при $f$, являющихся непрерывными 
функциями в $B(E)^{+}$. Процесс $X$ называется мерозначным ветвящимся прочессом (МВ-процессом), если его переходная полугруппа удовлетворяет соотношению

$$
\int_{M(E)} e^{-\nu(f)} Q_{r, t}(\mu, d \nu)=\exp \left\{-\mu\left(V_{r, t} f\right)\right\}, \quad r \leqslant t, f \in B(E)^{+} .
$$

При этом предположении мера $Q_{r, t}(\mu, \cdot)$ является безгранично делимой и $\left(V_{r, t}\right)_{r \leqslant t}$ образуют семейство операторов на $B(E)^{+}$, удовлетворяющих условию $V_{r, s} V_{s, t}=V_{r, t}$ для всех $r \leqslant s \leqslant t$. Это семейство называется полугруппой кумулянтов процесса $X$ (см., например, [34] и [35]). $(\xi, \phi)$-суперпроцесс, определенный во введении, является частным случаем MB-процесса.

Предположим, что $\left(Q_{r, t}\right)_{r \leqslant t}$ - переходная полугруппа МВ-процесса и $\left(N_{r, t}\right)_{r \leqslant t}$ - семейство вероятностных мер на $M(E)$. Назовем $\left(N_{r, t}\right)_{r \leqslant t}$ косой сверточной полугруппой (SC-полугруппой), ассоциированной с $\left(Q_{r, t}\right)_{r \leqslant t}$, если

$$
N_{r, t}=\left(N_{r, s} Q_{s, t}\right) * N_{s, t}, \quad r \leqslant s \leqslant t .
$$

Теорема 2.1. Соотношение (2.5) имеет место тогда и только mогда, когда

$$
Q_{r, t}^{N}(\mu, \cdot):=Q_{r, t}(\mu, \cdot) * N_{r, t}, \quad r \leqslant t, \mu \in M(E),
$$

определяет марковскую полугруппу $\left(Q_{r, t}^{N}\right)_{r \leqslant t}$ на $M(E)$.

Д ок а з а т е л ь с т в о. Пусть $\left(Q_{r, t}\right)_{r \leqslant t}$ и $\left(Q_{r, t}^{N}\right)_{r \leqslant t}$ заданы соотношениями (2.4) и (2.6) соответственно. Легко проверить, что (2.5) эквивалентно уравнению Чепмена-Колмогорова

$$
Q_{r, t}^{N}(\mu, \cdot)=\int_{M(E)} Q_{r, s}^{N}(\mu, d \nu) Q_{s, t}^{N}(\nu, \cdot), \quad r \leqslant s \leqslant t, \mu \in M(E),
$$

откуда вытекает требуемое утверждение.

Пусть $T$ - интервал и $\left(N_{r, t}\right)_{r \leqslant t}-\mathrm{SC}$-полугруппа, ассоциированная с $\left(Q_{r, t}\right)_{r \leqslant t}$. Если $\{Y: t \in T\}-$ марковский процесс с переходной полугруппой $\left(Q_{r, t}^{N}\right)_{r \leqslant t}$, определяемой соотношением (2.6), то мы будем называть его прочессом с иммиграчией, ассоциированным с $X$. (Конечно, $\mathrm{SC}$-полугруппы и процессы с иммиграцией могут также быть определены для некоторых более общих классов марковских процессов, фазовые пространства которых обладают полугрупповой структурой.)

Известно, что на $E$ можно задать метрику $\varrho$ так, что борелевская $\sigma$-алгебра на $E$, порожденная $\varrho$, будет совпадать с исходной борелевской $\sigma$-алгеброй, см., например, [2, с. 275]. Для множества $M(E)$, наделенного топологией слабой сходимости на $(E, \varrho)$, мы будем использовать обозначение $M\left(E_{\varrho}\right)$. Тогда $M\left(E_{\varrho}\right)$ локально компактно и метризуемо. Пусть $D\left(E_{\varrho}\right)^{+}$- счетное всюду плотное подмножество пространства строго положительных непрерывных функций на $(E, \varrho)$. 
Лемма 2.1. Пусть $\left\{F_{n}: n=1,2, \ldots\right\}$ - последовательность вероятностных мер на $M(E)$. Если существует предел

$$
L(f):=\lim _{n \rightarrow \infty} L_{F_{n}}(f), \quad f \in D\left(E_{\varrho}\right)^{+},
$$

$u L(f) \rightarrow 1$ при $f \rightarrow 0$, то существует вероятностная мера $F$ на $M(E)$ такая, ито $L_{F}(f)=L(f)$ для всех $f \in D\left(E_{\varrho}\right)^{+}$. При этом если каждая $F_{n}$ безгранично делима, то $F$ также обладает этим свойством.

Д ок аз а т е л с т в о. Пусть $\bar{M}\left(E_{\varrho}\right):=M\left(E_{\varrho}\right) \cup\{\infty\}$ обозначает одноточечное компактное расширение множества $M\left(E_{\varrho}\right)$. Тогда последовательность $\left\{F_{n}\right\}$, рассматриваемая как последовательность вероятностных мер на $\bar{M}\left(E_{\varrho}\right)$, относительно компактна. Пусть $\left\{F_{n_{k}}\right\}-$ подпоследовательность последовательности $\left\{F_{n}\right\}$, сходящаяся к некоторой вероятностной мере $F$ на $\bar{M}\left(E_{\varrho}\right)$. В силу $(2.7)$ и ограниченной сходимости мы имеем

$$
L(f)=\int_{\bar{M}\left(E_{\varrho}\right)} e^{-\nu(f)} F(d \nu), \quad f \in D\left(E_{\varrho}\right)^{+},
$$

где подынтегральное выражение доопределяется по непрерывности нулем в $\infty$. Так как $L(f) \rightarrow 1$ при $f \rightarrow 0$, то $F\left(M\left(E_{\varrho}\right)\right)=1$, и, следовательно, мы получаем первое утверждение. Второе утверждение доказывается непосредственным образом.

Для любого $\alpha \in[-\infty, \infty)$ семейство $\sigma$-конечных мер $\left(K_{t}\right)_{t>\alpha}$ на $M(E)$ называется входным законом (в $\alpha)$ для полугруппы $\left(Q_{t}\right)_{r \leqslant t}$, если $K_{r} Q_{r, t}=K_{t}$ для всех $t>r>\alpha$. Оно называется входным вероятностныим законом, если каждая $K_{t}$ является вероятностной мерой. Входной закон $\left(K_{t}\right)_{t>\alpha}$ называется замыкаемым, если существует $\sigma$-конечная мера $K_{\alpha}$ на $M(E)$ такая, что $K_{t}=K_{\alpha} Q_{\alpha, t}$ для всех $t>\alpha$. В этом случае $\left(K_{t}\right)_{t \geqslant \alpha}$ называется замкнутым входным законом для $\left(Q_{r, t}\right)_{r \leqslant t}$. Входной закон $\left(K_{t}\right)_{t>\alpha}$ называется минимальныцм, если каждый входной закон, доминируемый законом $\left(K_{t}\right)_{t>\alpha}$, пропорционален ему. Эти определения применимы к общим переходным полугруппам с очевидными модификациями.

П р и м е р 2.1. Пусть $T_{1} \subset \mathbf{R}-$ счетное множество и $\left\{\left(K_{s, t}\right)_{t>s}\right.$ : $\left.s \in T_{1}\right\}$ - семейство входных вероятностных законов для $\left(Q_{r, t}\right)_{r \leqslant t}$. Предположим, что

$$
-\sum_{s \in[r, t) \cap T_{1}} \ln L_{K_{s, t}}(1)<\infty
$$

для всех $r \leqslant t \in \mathbf{R}$. В силу леммы 2.1 мы можем заметить, что соотношение

$$
\ln L_{N_{r, t}}(f)=\sum_{s \in[r, t) \cap T_{1}} \ln L_{K_{s, t}}(f), \quad r \leqslant t, f \in B(E)^{+},
$$

определяет вероятностную меру $N_{r, t}$ на $M(E)$. Несложно проверить, что $\left(N_{r, t}\right)_{r \leqslant t}$ образует SC-полугруппу. Предположим, что для каждого $s \in T_{1}$ задан марковский процесс $\left(X_{s, t}\right)_{t>s}$ с переходной полугруппой 
$\left(Q_{r, t}\right)_{r \leqslant t}$ и одномерными распределениями $\left(K_{s, t}\right)_{t>s}$ и что элементы семейства $\left\{\left(X_{s, t}\right)_{t>s}: s \in T_{1}\right\}$ независимы. Тогда для любого $t \geqslant \alpha$ случайная мера $Y_{t}:=\sum_{s \in[\alpha, t) \cap T_{1}} X_{s, t}$ почти наверное корректно определена и $\left\{Y_{t}: t \geqslant \alpha\right\}$ - процесс с иммиграцией, соответствующий SC-полугруппе, задаваемой соотношением (2.8).

П р и м е р 2.2. Пусть $T_{2} \subset \mathbf{R}-$ счетное множество и $\left\{\left(K_{s, t}\right)_{t \geqslant s}\right.$ : $\left.s \in T_{2}\right\}$ - семейство замкнутых входных вероятностных законов для $\left(Q_{r, t}\right)_{r \leqslant t}$ таких, что $-\sum_{s \in(r, t] \cap T_{2}} \ln L_{K_{s, t}}(1)<\infty$ для всех $r \leqslant t \in \mathbf{R}$. Тогда можно определить $\mathrm{SC}$-полугруппу $\left(N_{r, t}\right)_{r \leqslant t}$ с помошью соотношения

$$
\ln L_{N_{r, t}}(f)=\sum_{s \in(r, t] \cap T_{2}} \ln L_{K_{s, t}}(f), \quad r \leqslant t, f \in B(E)^{+} .
$$

Соответствующий процесс с иммиграцией может быть построен так же, как в предыдущем примере.

П р и м е р 2.3. Предположим, что $\left(N_{r, t}\right)_{r \leqslant t}-$ семейство вероятностных мер на $M(E)$, задаваемое соотношением

$$
\ln L_{N_{r, t}}(f)=\int_{r}^{t} \ln L_{K_{s, t}}(f) \zeta(d s), \quad r \leqslant t, f \in B(E)^{+},
$$

где $\zeta(d s)$ - радонова мера на $\mathbf{R}$ и $\left\{\left(K_{s, t}\right)_{t>s}: s \in \mathbf{R}\right\}$ - семейство безгранично делимых входных вероятностных законов для $\left(Q_{r, t}\right)_{r \leqslant t}$. Тогда $\left(N_{r, t}\right)_{r \leqslant t}$ является SC-полугруппой.

П р и м е р 2.4. Предположим, что имеется вероятностное пространство, на котором заданы два процесса $\left\{X_{t}^{1}: t \geqslant 0\right\}$ и $\left\{X_{t}^{2}: t \geqslant 0\right\}$, где $\left\{X_{t}^{1}: t \geqslant 0\right\}$ - суперпроцесс с параметрами $\left(\xi_{1}, \phi_{1}\right)$, a $\left\{X_{t}^{2}: t \geqslant 0\right\}$ при условии $\left\{X_{t}^{1}: t \geqslant 0\right\}$ является процессом с иммиграцией, соответствующим SC-полугруппе, задаваемой соотношением

$$
\ln L_{N_{r, t}}(f)=-\int_{r}^{t} X_{s}\left(V_{t-s}^{2} f\right) d s, \quad r \leqslant t, f \in B(E)^{+},
$$

где $\left(V_{t}^{2}\right)_{t \geqslant 0}$ определяется соотношением (1.2) с параметрами $\left(\xi_{2}, \phi_{2}\right)$. Тогда $\left\{\left(X_{t}^{1}, X_{t}^{2}\right): t \geqslant 0\right\}$ также является марковским процессом. Интуитивно ясно, что он описывает эволюцию популяции с двумя типами «частиц» на $E$, при которой частицы второго типа могут порождаться частицами обоих типов, см. [18]. Более общие формы суперпроцессов с несколькими типами частиц изучались в $[16],[17],[23]$ и др.

3. Разложение косых сверточных полугрупп. В этом пункте мы докажем теорему о разложении для неоднородной SC-полугруппы, которая используется в конструкции однородного процесса с иммиграцией. Рассмотрим переходную полугруппу $\left(Q_{r, t}\right)_{r \leqslant t}$ MB-процесса, определенного в предыдущем пункте.

Теорема 3.1. Семейство вероятностных мер $\left(N_{r, t}\right)_{r \leqslant t}$ на $M(E)$ образует $S C$-полугруппу, ассочиированную $c\left(Q_{r, t}\right)_{r \leqslant t}$, тогда и только 
тогда, когда

$$
\begin{aligned}
\ln L_{N_{r, t}}(f)= & \sum_{s \in[r, t) \cap T_{1}} \ln L_{K_{s, t}^{1}}(f)+\sum_{s \in(r, t] \cap T_{2}} \ln L_{K_{s, t}^{2}}(f) \\
& +\int_{r}^{t} \ln L_{K_{s, t}^{3}}(f) \zeta(d s), \quad r \leqslant t, f \in B(E)^{+},
\end{aligned}
$$

где $T_{1}, T_{2} \subset \mathbf{R}$ - счетные множества, $\zeta(d s)$ - рассеянная радонова мера на $\mathbf{R},\left\{\left(K_{s, t}^{1}\right)_{t>s}: s \in T_{1}\right\}-$ семейство входных вероятностных законов, $\left\{\left(K_{s, t}^{2}\right)_{t \geqslant s}: s \in T_{2}\right\}-$ семейство замкнутых входных вероятностных законов, $a\left\{\left(K_{s, t}^{3}\right)_{t>s}: s \in \mathbf{R}\right\}-$ семейство безгранично делимых входных вероятностных законов для $\left(Q_{r, t}\right)_{r \leqslant t}$.

В принципе, входные законы могут быть получены путем применения леммы 2.1. Доказательство довольно утомительно, поскольку мы не предполагаем наличия феллеровского свойства и класс $D\left(E_{\varrho}\right)^{+}$не сохраняется полугруппой кумулянтов $\left(V_{r, t}\right)_{r \leqslant t}$. Разобьем доказательство на несколько лемм. Предположим, что $\left(N_{r, t}\right)_{r \leqslant t}-\mathrm{SC}$-полугруппа, ассоциированная с $\left(Q_{r, t}\right)_{r \leqslant t}$, и пусть

$$
J_{r, t}(f)=-\ln \int_{M(E)} e^{-\nu(f)} N_{r, t}(d \nu), \quad r \leqslant t, f \in B(E)^{+} .
$$

Тогда соотношение (2.5) эквивалентно соотношению

$$
J_{r, t}(f)=J_{r, s}\left(V_{s, t} f\right)+J_{s, t}(f), \quad r \leqslant s \leqslant t, f \in B(E)^{+} .
$$

Из (3.3) видно, что $J_{r, t}(f)$ - невозрастающая функция от $r \leqslant t$ и $J_{t, t}(f)=0$. Тогда существует радонова мера $G_{t}(f, \cdot)$ на $(-\infty, t]$ такая, что $G_{t}(f,\{t\})=\lim _{r \uparrow t} J_{r, t}(f)$ и при $r \leqslant s<t$.

$$
G_{t}(f,(r, s])=\lim _{v \downarrow s} \lim _{u \downarrow r}\left[J_{u, t}(f)-J_{v, t}(f)\right]=\lim _{v \downarrow s} \lim _{u \downarrow r} J_{u, v}\left(V_{v, t} f\right) .
$$

Лемма 3.1. (i) Ecли $f, g \in B(E)^{+} u 0 \leqslant g \leqslant c f$ с некоторой конcтантой $c \geqslant 1$, mo $G_{t}(g, \cdot) \leqslant c G_{t}(f, \cdot)$.

(ii) Для $t \leqslant u$ u $f \in B(E)^{+}$имеет место соотношение $G_{u}(f, d s)=$ $G_{t}\left(V_{t, u} f, d s\right) н a(-\infty, r)$.

(iii) Для $t \leqslant u$ u $f \in B(E)^{+}$имеет место соотношение $G_{u}(f, d s) \ll$ $G_{t}(1, d s) н a(-\infty, t)$.

Д ок а з а т е л ь с т в о. При $r \leqslant s<t \leqslant u$ воспользуемся (2.4), (3.2) и неравенством Иенсена, чтобы убедиться, что

$$
\begin{aligned}
G_{t}(g,(r, s]) & =\lim _{v \downarrow s} \lim _{w \downarrow r} J_{w, v}\left(V_{v, t} g\right) \leqslant \lim _{v \downarrow s} \lim _{w \downarrow r} J_{w, v}\left(V_{v, t}(c f)\right) \\
& \leqslant c \lim _{v \downarrow s} \lim _{w \downarrow r} J_{w, v}\left(V_{v, t} f\right)=c G_{t}(f,(r, s]) .
\end{aligned}
$$

Аналогично, мы имеем $G_{t}(g,\{t\}) \leqslant c G_{t}(f,\{t\})$. Тем самым, п. (i) доказан. Пусть $r \leqslant s<t \leqslant u$. В силу (3.4) и полугруппового свойства $\left(V_{r, t}\right)_{r \leqslant t}$ мы имеем

$$
G_{u}(f,(r, s])=\lim _{v \downarrow s} \lim _{w \downarrow r} J_{w, v}\left(V_{v, t} V_{t, u} f\right)=G_{t}\left(V_{t, u} f,(r, s]\right),
$$


откуда вытекает (ii). Комбинируя (i) и (ii), получаем

$$
G_{u}(f,(r, s])=G_{t}\left(V_{t, u} f,(r, s]\right) \leqslant\left(\left\|V_{t, u} f\right\|+1\right) G_{t}(1,(r, s]),
$$

откуда вытекает (iii).

Ясно, что имеет место единственное разложение

$$
J_{r, t}(f)=J_{r, t}^{1}(f)+J_{r, t}^{2}(f)+J_{r, t}^{3}(f), \quad r \leqslant t, f \in B(E)^{+},
$$

где $J_{r, t}^{1}(f)$ - непрерывная слева невозрастающая ступенчатая функция, $J_{r, t}^{2}(f)$ - непрерывная справа невозрастающая ступенчатая функция и $J_{r, t}^{3}(f)$ - непрерывная невозрастающая функция от $r \leqslant t$, причем $J_{t, t}^{i}(f)=0$ для $i=1,2,3$. В силу единственности, $\left(J_{r, t}^{i}\right)_{r \leqslant t}$ также удовлетворяет соотношению (3.3). Применяя лемму 2.1, мы можем получить

$$
N_{r, t}=N_{r, t}^{1} * N_{r, t}^{2} * N_{r, t}^{3}, \quad r \leqslant t
$$

где $\left(N_{r, t}^{i}\right)_{r \leqslant t}$ - SC-полугруппа, соответствуюшая функционалу $\left(J_{r, t}^{i}\right)_{r \leqslant t}$. Заметим также, что $J_{s, t}^{1}(f)$ и $J_{s, t}^{2}(f)$ задают, соответственно, атомические меры $G_{t}^{1}(f, \cdot)$ и $G_{t}^{2}(f, \cdot)$ на $(-\infty, t]$, а $J_{s, t}^{3}(f)$ задает рассеянную меpy $G_{t}^{3}(f, \cdot)$ на $(-\infty, t]$.

Лемма 3.2. Существуют счетные множества $T_{1}, T_{2} \subset \mathbf{R}$, входные вероятностные захоны $\left\{\left(K_{r, t}^{1}\right)_{t>r}: r \in T_{1}\right\}$ и замкнутые входные вероятностные законы $\left\{\left(K_{r, t}^{2}\right)_{t \geqslant r}: r \in T_{2}\right\}$ такие, ито

$$
\begin{aligned}
& J_{r, t}^{1}(f)=-\sum_{s \in[r, t) \cap T_{1}} \ln L_{K_{s, t}^{1}}(f), \quad r \leqslant t, f \in B(E)^{+}, \\
& J_{r, t}^{2}(f)=-\sum_{s \in(r, t] \cap T_{2}} \ln L_{K_{s, t}^{2}}(f), \quad r \leqslant t, f \in B(E)^{+} .
\end{aligned}
$$

Д ок а з т е л с т в о. Поскольку рассуждения аналогичны, мы приведем лишь доказательство (3.8). Напомним, что $J_{s, t}^{2}(f)$ непрерывна справа по $s \leqslant t$. Следовательно,

$$
G_{t}^{2}(f,\{s\})=\downarrow \lim _{r \uparrow s}\left[J_{r, t}^{2}(f)-J_{s, t}^{2}(f)\right]=\downarrow \lim _{r \uparrow s} J_{r, s}^{2}\left(V_{s, t} f\right),
$$

$f \in B(E)^{+}$. Таким образом,

$$
\exp \left\{-G_{t}^{2}(f,\{s\})\right\}=\uparrow \lim _{r \uparrow s} \int_{M(E)} e^{-\nu(f)} N_{r, s}^{2} Q_{s, t}(d \nu), \quad f \in B(E)^{+} .
$$

В силу леммы 2.1 мы замечаем, что

$$
\exp \left\{-G_{t}^{2}(f,\{s\})\right\}=\int_{M(E)} e^{-\nu(f)} K_{s, t}^{2}(d \nu), \quad f \in D\left(E_{\varrho}\right)^{+},
$$

для вероятностной меры $K_{s, t}^{2}$ на $M(E)$. Пусть $Q=\left\{u_{i}: i=1,2, \ldots\right\}-$ счетное всюду плотное подмножество $\mathbf{R}$, и пусть $T_{2}$ - множество атомов мер $\left\{G_{u_{i}}^{2}(1, \cdot): i=1,2, \ldots\right\}$. В силу (3.10) и леммы 3.1 мы получаем

$$
\ln L_{N_{r, t}^{2}}(f)=-G_{t}^{2}(f,(r, t])=\ln L_{K_{t, t}^{2}}(f)+\sum_{s \in(r, t) \cap T_{2}} \ln L_{K_{s, t}^{2}}(f), \quad r \leqslant t,
$$


сначала для $f \in D\left(E_{\varrho}\right)^{+}$, а затем для всех $f \in B(E)^{+}$. В частности, (3.10) также имеет место для $f \in B(E)^{+}$. Но из (3.9) вытекает, что $G_{t}^{2}(f,\{s\})=G_{s}^{2}\left(V_{s, t} f,\{s\}\right)$. Тогда $K_{s, s} Q_{s, t}=K_{s, t}$ при $s \leqslant t$, т.е. $\left(K_{s, t}\right)_{t \geqslant s}$ образует замкнутый входной закон. Если $-\ln L_{K_{t, t}^{2}}(f)>0$, то $-\ln L_{K_{t, t}^{2}}(1)>0$. В силу непрерывности $V_{t, u} 1$ по $u \geqslant t$ мы имеем

$$
G_{u}^{2}(1,\{t\})=-\ln L_{K_{t, u}^{2}}(1)=-\ln L_{K_{t, t}^{2}}\left(V_{t, u} 1\right)>0
$$

для некоторого $u \in Q$. Тогда $t \in Q$, и, следовательно, мы получаем (3.8).

Лемма 3.3. Существует рассеянная радонова мера $\zeta(d s)$ на $\mathbf{R}$ maxas, чmо $G_{t}^{3}(f, d s) \ll \zeta(d s)$ длs всех $t \in \mathbf{R} u f \in B(E)^{+}$.

Доказ а тельст во. Пусть $Q=\left\{u_{i}: i=1,2, \ldots\right\}$ - счетное всюду плотное подмножество $\mathbf{R}$. Выберем $a_{i}>0$ так, чтобы $\sum_{i=1}^{\infty} a_{i} G_{u_{i}}^{3}\left(1,\left(u_{i}-1, u_{i}\right]\right)<\infty$. Мы можем определить рассеянную радонову (на самом деле конечную) меру $\zeta$ на $\mathbf{R}$ с помощью соотношения

$$
\zeta(d s)=\sum_{i=1}^{\infty} 1_{\left(u_{i}-1, u_{i}\right]}(s) G_{u_{i}}^{3}(1, d s), \quad s \in \mathbf{R} .
$$

Если $G_{t}^{3}(f, B)>0$ для борелевского множества $B \subset(-\infty, t]$, то $G_{t}^{3}\left(f,\left(u_{i}-1, u_{i}\right) \cap B\right)>0$ для некоторого $u_{i} \in(-\infty, t) \cap Q$. Поэтому из леммы 3.1 вытекает, что $G_{u_{i}}^{3}\left(1,\left(u_{i}-1, u_{i}\right) \cap B\right)>0$, и, следовательно, $\zeta(B)>0$.

Лемма 3.4. Производная Радона-Никодима $G_{t}^{3}(f, d s) / \zeta(d s)$ имеет версию $D_{s, t}(f)$ с представлением

$$
D_{s, t}(f)=-\ln L_{F_{s, t}}(f), \quad s<t, f \in B(E)^{+},
$$

где $F_{s, t}$ - безгранично делимая вероятностная мера на $M(E)$.

Д ок аз а т л в с т о. Пусть $r<t$. Чтобы избежать тривиальных случаев, предположим, что $\zeta(r, t]>0$. Возьмем возрастающую последовательность упорядоченных множеств $\pi_{n}=\left\{s_{0}^{n}, s_{1}^{n}, \ldots, s_{m(n)}^{n}\right\}$, такую, что $r=s_{0}^{n}<s_{0}^{n}<\cdots<s_{m(n)}^{n}=t$ и $\lim _{n \rightarrow \infty} \delta_{n}=0$, где $\delta_{n}=\max \left\{s_{i}^{n}-s_{i-1}^{n}: 1 \leqslant i \leqslant m(n)\right\}$. Пусть $\mathscr{F}_{n}-\sigma$-алгебра на $(r, t]$, порожденная интервалами $\left(s_{0}^{n}, s_{1}^{n}\right], \ldots,\left(s_{m(n)-1}^{n}, s_{m(n)}^{n}\right]$, и пусть

$$
M_{n}(f)(s)= \begin{cases}\frac{G_{t}^{3}\left(f,\left(s_{i-1}^{n}, s_{i}^{n}\right]\right)}{\zeta\left(s_{i-1}^{n}, s_{i}^{n}\right]}, & \text { если } s_{i-1}^{n}<s \leqslant s_{i}^{n} \text { и } \zeta\left(s_{i-1}^{n}, s_{i}^{n}\right]>0, \\ 0, & \text { если } s_{i-1}^{n}<s \leqslant s_{i}^{n} \text { и } \zeta\left(s_{i-1}^{n}, s_{i}^{n}\right]=0 .\end{cases}
$$

Тогда относительно вероятностной меры $(\zeta(r, t])^{-1} \zeta$ последовательность $\left\{M_{n}(f), \mathscr{F}_{n}: n \geqslant 1\right\}$ является мартингалом, замкнутым справа производной Радона-Никодима $G_{t}^{3}(f, d s) / \zeta(d s)$. Но так как $\left\{\mathscr{F}_{n}: n \geqslant 1\right\}$ порождает борелевскую $\sigma$-алгебру на $(r, t]$, то $M_{n}(f)(s)$ сходится при $n \rightarrow \infty$ к 
$G_{t}^{3}(f, d s) / \zeta(d s)$ для $\zeta$-почти всех $s \in(r, t]$ по теореме о сходимости мартингалов. Тогда можно взять множество $A_{t} \subset(-\infty, t]$ полной $\zeta$-меры такое, что для любого $s \in A_{t}$ сушествуют последовательности $r_{k} \uparrow s$ и $s_{k} \downarrow s$, удовлетворяющие

$$
\frac{G_{t}^{3}(f, d s)}{\zeta(d s)}=\lim _{k \rightarrow \infty} \frac{G_{t}^{3}\left(f,\left(r_{k}, s_{k}\right]\right)}{\zeta\left(r_{k}, s_{k}\right]}, \quad f \in D\left(E_{\varrho}\right)^{+} .
$$

Ясно, что $G_{t}^{3}\left(f,\left(r_{k}, s_{k}\right]\right)=G_{s_{k}}\left(V_{s_{k}, t} f,\left(r_{k}, s_{k}\right]\right)$ стремится к нулю при $k \rightarrow \infty$. Следовательно,

$$
\begin{aligned}
\frac{G_{t}^{3}(f, d s)}{\zeta(d s)} & =\lim _{k \rightarrow \infty} \frac{1-\exp \left\{-G_{s_{k}}\left(V_{s_{k}, t} f,\left(r_{k}, s_{k}\right]\right)\right\}}{\zeta\left(r_{k}, s_{k}\right]} \\
& =\lim _{k \rightarrow \infty} \frac{1}{\zeta\left(r_{k}, s_{k}\right]} \int_{M(E)^{\circ}}\left(1-e^{-\nu(f)}\right) N_{r_{k}, s_{k}} Q_{s_{k}, t}(d \nu)
\end{aligned}
$$

для $f \in D\left(E_{\varrho}\right)^{+}$. С другой стороны, поскольку

$$
\int_{r}^{t} \frac{G_{t}^{3}(f, d s)}{\zeta(d s)} \zeta(d s)=-\ln \int_{M(E)} e^{-\nu(f)} N_{r, t}^{3}(d \nu),
$$

выбирая меньшее множество $A_{t} \subset(-\infty, t]$ полной $\zeta$-меры, можно получить, что $G_{t}^{3}(f, d s) / \zeta(d s) \downarrow 0$ при $f \downarrow 0$ для всех $s \in A_{t}$. В силу (3.13) и леммы 2.1 существуют безгранично делимые вероятностные меры $\left\{F_{s, t}: s \in A_{t}\right\}$ такие, что

$$
\frac{G_{t}^{3}(f, d s)}{\zeta(d s)}=-\ln L_{F_{s, t}}(f), \quad s \in A_{t}, f \in D\left(E_{\varrho}\right)^{+} .
$$

Полагая $F_{s, t}=\delta_{0}$ при $s \in(-\infty, t] \backslash A_{t}$, мы получаем

$$
G_{t}^{3}(f,(r, t])=-\ln \int_{M(E)} e^{-\nu(f)} N_{r, t}^{3}(d \nu)=-\int_{r}^{t} \ln L_{F_{s, t}}(f) \zeta(d s)
$$

сначала для $f \in D\left(E_{\varrho}\right)^{+}$, а затем для всех $f \in B(E)^{+}$.

Лемма 3.5. Для полугруппы $\left(Q_{r, t}\right)_{r \leqslant t}$ существуют безграниино делимые входные вероятностные законы $\left\{\left(K_{s, t}\right)_{t>s}: s \in \mathbf{R}\right\}$ такие, ито

$$
J_{r, t}^{3}(f)=-\int_{r}^{t} \ln L_{K_{s, t}}(f) \zeta(d s), \quad t \geqslant r, f \in B(E)^{+} .
$$

Д ок а з а т е льст в о. По лемме 3.1 мы имеем $G_{t}^{3}(f, d s)=$ $G_{r}^{3}\left(V_{r, t} f, d s\right)$ при $s<r \leqslant t$. Отсюда вытекает, что $D_{s, t}(f)=D_{s, r}\left(V_{r, t} f\right)$ для $\zeta$-почти всех $s \in(-\infty, r]$. Пусть $\lambda-$ мера Лебега на $\mathbf{R}$. По лемме Фубини сушествует множество $B(f) \subseteq \mathbf{R}$ полной $\zeta$-меры и множества $C_{s}(f) \subseteq[s, \infty)$ и $C_{s, r}(f) \subseteq[r, \infty)$ полной $\lambda$-меры такие, что

$$
D_{s, t}(f)=D_{s, r}\left(V_{r, t} f\right), \quad s \in B(f), r \in C_{s}(f), t \in C_{s, r}(f) \text {. . }
$$

По лемме $3.4 D_{s, t}$ и $D_{s, r} \circ V_{r, t}$ определяются их сужениями на счетный класс $D\left(E_{\varrho}\right)^{+}$. Тогда для множества $B \subseteq \mathbf{R}$ полной $\zeta$-меры и множеств $C_{s} \subseteq(s, \infty)$ и $C_{s, r} \subseteq(r, \infty)$ полной $\lambda$-меры мы имеем

$$
D_{s, t}=D_{s, r} \circ V_{r, t}, \quad s \in B, r \in C_{s}, t \in C_{s, r},
$$


как операторы на $B(E)^{+}$. Для любого $s \in B$ выберем последовательность $\left\{s_{k}\right\} \subset C_{s}$ с $s_{k} \downarrow s$. Согласно (3.15), мы получаем

$$
D_{s, s_{k}} \circ V_{s_{k}, t}=D_{s, s_{k+1}} \circ V_{s_{k+1}, t}=D_{s, t}, \quad t \in C_{s, s_{k}} \cap C_{s, s_{k+1}} .
$$

В силу леммы 3.4 и наших предположений $D_{s, s_{k}}\left(V_{s_{k}, t} f\right)$ и $D_{s, s_{k+1}}\left(V_{s_{k+1}, t} f\right)$ непрерывны справа по $t \geqslant s_{k+1}$ при $f \in C(E)^{+}$. Тогда мы имеем

$$
D_{s, s_{k}} \circ V_{s_{k}, t}=D_{s, s_{k+1}} \circ V_{s_{k+1}, t}, \quad t \geqslant s_{k},
$$

как операторы на $B(E)^{+}$. Для $t>s$ возьмем некоторое $s_{k} \in(s, t)$ и положим $I_{s, t}=D_{s, s_{k}} \circ V_{s_{k}, t}, I_{s, t}$ не зависит от выбора $s_{k}$ в силу (3.16). Соответственно, пусть $F_{s, s_{k}}$ - безгранично делимая вероятностная мера на $M(E)$, определяемая леммой 3.4 , и пусть $K_{s, t}=F_{s, s_{k}} Q_{s_{k}, t}, K_{s, t}$ также не зависит от выбора $s_{k}$. Ясно, что $\left(K_{s, t}\right)_{t>s}$ образует входной закон для $\left(Q_{r, t}\right)_{r \leqslant t}$ и

$$
I_{s, t}=-\ln L_{F_{s, s_{k}}} \circ V_{s_{k}, t}=-\ln L_{K_{s, t}}(f), \quad t>s .
$$

Из (3.15) мы имеем $I_{s, t}=D_{s, t}$ для $\lambda$-почти всех $t \in(s, \infty)$. Если $s \in$ $\mathbf{R} \backslash B$, то мы положим $I_{s, t}=0$ для всех $t>s$. Тогда лемма Фубини обеспечивает существование множества $U \subseteq \mathbf{R}$ полной $\lambda$-меры такого, что для любого $t \in U$ мы имеем $I_{s, t}=D_{s, t}$ для $\zeta$-почти всех $s \in(-\infty, t)$. Следовательно, если $t \in U$, то

$$
J_{r, t}^{3}(f)=G_{t}^{3}(f,(r, t])=\int_{r}^{t} I_{s, t}(f) \zeta(d s), \quad r \leqslant t, f \in B(E)^{+} .
$$

Напомним, что и $\zeta$, и $G_{t}^{3}(f, \cdot)$ являются рассеянными мерами. Для $t \in$ $\mathbf{R} \backslash U$ выберем последовательность $\left\{t_{k}\right\} \subset U$ такую, что $t_{k} \uparrow t$. По (3.18) и лемме 3.1 мы имеем

$$
\begin{aligned}
J_{r, t}^{3}(f) & =\lim _{k \rightarrow \infty} G_{t}^{3}\left(f,\left(r, t_{k}\right]\right)=\lim _{k \rightarrow \infty} G_{t_{k}}^{3}\left(V_{t_{k}, t} f,\left(r, t_{k}\right]\right) \\
& =\lim _{k \rightarrow \infty} \int_{r}^{t_{k}} I_{s, t_{k}}\left(V_{t_{k}, t} f\right) \zeta(d s)=\lim _{k \rightarrow \infty} \int_{r}^{t_{k}} I_{s, t}(f) \zeta(d s) \\
& =\int_{r}^{t} I_{s, t}(f) \zeta(d s),
\end{aligned}
$$

откуда вытекает требуемый результат.

Д ок азател в с т о т е о е мы 3.1. Легко видеть, что для семейства $\left(N_{r, t}\right)_{r \leqslant t}$, определенного соотношением (3.1), имеет место (2.5). Комбинируя (3.5) и леммы 3.2 и 3.5 , мы замечаем, что любая SC-полугруппа допускает разложение (3.1).

Пусть $\left(Q_{t}\right)_{t \geqslant 0}$ - переходная полугруппа однородного МВ-процесса. Следующий частный случай теоремы 3.1 был доказан в [25].

Теорема 3.2 (см. [25]). Семейство вероятностных мер $\left(N_{t}\right)_{t \geqslant 0}$ на $M(E)$ является $S C$-полугруппой, ассочиированной $c\left(Q_{t}\right)_{t \geqslant 0}$, тогда 
$u$ только тогда, когда для $\left(Q_{t}\right)_{t \geqslant 0}$ существует безгранично делимый вероятностный входной закон $\left(K_{t}\right)_{t>0}$ такой, что

$$
\ln L_{N_{t}}(f)=\int_{0}^{t}\left[\ln L_{K_{s}}(f)\right] d s, \quad t \geqslant 0, f \in B(E)^{+} .
$$

4. Построение процесса с иммиграцией. Чтобы наилучшим образом использовать существующую литературу, в этом и следующих пунктах мы ограничимся рассмотрением переходной полугруппы $\left(Q_{t}\right)_{t \geqslant 0}$ однородного правого борелевского МВ-процесса $X$. Пусть $\left(Q_{t}^{\circ}\right)_{t \geqslant 0}$ обозначает сужение $\left(Q_{t}\right)_{t \geqslant 0}$ на $M(E)^{\circ}$.

Сначала напомним некоторые факты из теории потенциала, см., например, [6] и [14]. Семейство $\sigma$-конечных мер $\left(J_{t}\right)_{t \in \mathbf{R}}$ называется правилом вхождения для $\left(Q_{t}^{\circ}\right)_{t \geqslant 0}$, если $J_{s} Q_{t-s}^{\circ} \leqslant J_{t}$ при $t>s t$, $s \in \mathbf{R}$ и $J_{s} Q_{t-s}^{\circ} \uparrow J_{t}$ при $s \uparrow t$. Заметим, что входной закон $\left(H_{t}\right)_{t>r}$ для $r \in[-\infty, \infty)$ может быть расширен до правила вхождения, если положить $H_{t}=0$ для $t \leqslant r$. Пусть $W(M(E))$ обозначает пространство траекторий $\left\{w_{t}: t \in \mathbf{R}\right\}$, которые являются $M(E)^{\circ}$-значными и непрерывными справа на открытом интервале $(\alpha(w), \beta(w))$ и принимают значение, равное нулевой мере, в других точках. Траектория [0], тождественно равная 0 , соответствует пустому интервалу $(\alpha, \beta)$. Положим $\alpha([0])=+\infty$ и $\beta([0])=-\infty$. Пусть $\left(\mathscr{G}^{\circ}, \mathscr{G}_{t}^{\circ}\right)_{t \in \mathbf{R}}-$ естественные $\sigma$-алгебры на $W(M(E))$, порожденные координатным процессом. Oператоры сдвига $\left\{\sigma_{t}: t \in \mathbf{R}\right\}$ на $W(M(E))$ определяются соотношением $\sigma_{t} w_{s}=w_{t+s}$. Каждому правилу вхождения $\left(J_{t}\right)_{t \in \mathbf{R}}$ соответствует единственная $\sigma$-конечная мера $\mathbf{Q}^{J}$ на $\left(W(M(E)), \mathscr{G}^{\circ}\right)$, относительно которой координатный процесс $\left\{w_{t}: t \in \mathbf{R}\right\}$ является марковским с одномерными распределениями $\left(J_{t}\right)_{t \in \mathbf{R}}$ и полугруппой $\left(Q_{t}^{\circ}\right)_{t g e 0}$. А именно,

$$
\begin{gathered}
\mathbf{Q}^{J}\left\{\alpha<t_{1}, w_{t_{1}} \in d \nu_{1}, w_{t_{2}} \in d \nu_{2}, \ldots, w_{t_{n}} \in d \nu_{n}, t_{n}<\beta\right\} \\
=J_{t_{1}}\left(d \nu_{1}\right) Q_{t_{2}-t_{1}}^{\circ}\left(\nu_{1}, d \nu_{2}\right) \cdots Q_{t_{n}-t_{n-1}}^{\circ}\left(\nu_{n-1}, d \nu_{n}\right)
\end{gathered}
$$

для всех $t_{1}<\cdots<t_{n} \in \mathbf{R}$ и $\nu_{1}, \ldots, \nu_{n} \in M(E)^{\circ}$. Существование этой меры было доказано С. Е. Кузнецовым [22], также см. [15]. Систему $\left(W(M(E)), \mathscr{G}^{\circ}, \mathscr{G}_{t}^{\circ}, w_{t}, \mathbf{Q}^{J}\right)$ обычно называют прочессом Кузнечова, определяемым $\left(J_{t}\right)_{t \in \mathbf{R}}, \mathbf{a} \mathbf{Q}^{J}$ называют мерой Кузнечова. Имеет место представление

$$
J_{t}=H_{-\infty, t}+\int_{\mathbf{R}} H_{s, t} \rho(d s), \quad t \in \mathbf{R},
$$

где $\rho(d s)$ - радонова мера на $\mathbf{R}$ и $\left(H_{s, t}\right)_{t \in \mathbf{R}}-$ входной закон при $s \in[-\infty, \infty)$. Из этого представления следует, что

$$
\mathbf{Q}^{J}(d w)=\mathbf{Q}_{-\infty}(d w)+\int_{\mathbf{R}} \mathbf{Q}_{s}(d w) \rho(d s)
$$


где $\mathbf{Q}_{s}(d w)$ - мера Кузнецова, определенная законом $\left(H_{s, t}\right)_{t \in \mathbf{R}}$, см. [15]. Если $\left(J_{t}\right)_{t \in \mathbf{R}}-$ входной закон при $r \in[-\infty, \infty)$, то носителем $\mathbf{Q}^{J}$ является $W_{r}(M(E))$ - подмножество множества $W(M(E))$, включающее В себя траектории $\left\{w_{t}: t \in \mathbf{R}\right\}$ такие, что $\alpha(w)=r$. В частности, если $F$ - избыточная мера для $\left(Q_{t}^{\circ}\right)_{t \geqslant 0}$ и $J_{t} \equiv F$, то $\mathbf{Q}^{J}$ стационарна, т.е. $\mathbf{Q}^{J} \circ \sigma_{t}^{-1}=\mathbf{Q}^{J}$ для всех $t \in \mathbf{R}$.

Теперь предположим, что $\left(J_{t}\right)_{t \in \mathbf{R}}-$ правило вхождения для $\left(Q_{t}^{\circ}\right)_{t \geqslant 0}$ с представлением (4.2) и $N^{J}(d w)$ - пуассоновская случайная мера на $W(M(E))$ с интенсивностью $\mathbf{Q}^{J}(d w)$. Легко видеть, что процесс

$$
Y_{t}^{J}:=\int_{W(M(E))} w_{t} N^{J}(d w)
$$

почти наверное корректно определен для каждого $t \in \mathbf{R}$.

Лемма 4.1. В ситуачии, описанной выше, $\left\{Y_{t}^{J}: t \in \mathbf{R}\right\}$ является прочессом с иммиграчией, соответствующим (неоднородной) $S C$-полугруппе $\left(N_{r, t}\right)_{r \leqslant t}$, определяемой соотночением

$$
\ln L_{N_{r, t}}(f)=-\int_{[r, t)} \int_{M(E)^{\circ}}\left(1-e^{-\nu(f)}\right) H_{s, t}(d \nu) \rho(d s),
$$

$r \leqslant t, f \in B(E)^{+}$.

Д ок а з а т е л ь с т в о. Согласно (4.3), для любой ограниченной борелевской функции $F$ на $M(E)$ такой, что $F(0)=0$, мы имеем

$$
\mathbf{Q}^{J}\left\{F\left(w_{t}\right) ; r \leqslant \alpha<t\right\}=\int_{[r, t)} H_{s, t}(F) \rho(d s) .
$$

Теперь требуемые результаты вытекают из (4.6) и марковского свойства $\mathbf{Q}^{J}$.

Пусть $\left(N_{t}\right)_{t \geqslant 0}-\mathrm{SC}$-полугруппа, ассоциированная с $\left(Q_{t}\right)_{t \geqslant 0}$, заданная соотношением (3.19). Предположим, что $K_{t}=I\left(\eta_{t}, H_{t}\right)$ и $N_{t}=$ $I\left(\gamma_{t}, G_{t}\right)$ при $t>0$ и $t \geqslant 0$ соответственно.

Лемма 4.2. Пусть $G_{t}=0$ при $t<0$. Тогда $\left(G_{t}\right)_{t \in \mathbf{R}}$ является правилом вхождения $л я_{\text {s }}\left(Q_{t}^{\circ}\right)_{t \geqslant 0}$.

Д ок а з а т е л в с т в о. Напомним, что $Q_{t}(\mu, \cdot)$ - безгранично делимая вероятностная мера на $M(E)$ при всех $t \geqslant 0$ и $\mu \in M(E)$. Предположим, что $Q_{t}\left(\delta_{x}, \cdot\right)=I\left(\lambda_{t}(x, \cdot), L_{t}(x, \cdot)\right)$. Согласно $(1.4)$,

$$
G_{t}=G_{t-r}+G_{r} Q_{t-r}^{\circ}+\int_{E} \gamma_{r}(d x) L_{t-r}(x, \cdot), \quad t>r>0,
$$

и, следовательно, $G_{r} Q_{t-r} \leqslant G_{t}$. Из (3.19) мы имеем

$$
G_{t} Q_{t-r}^{\circ}=\int_{0}^{t} H_{s} Q_{t-r}^{\circ} d s=G_{r} Q_{t-r}^{\circ}+\int_{r}^{t} H_{s} Q_{t-r}^{\circ} d s,
$$

так что $G_{r} Q_{t-r}^{\circ} \uparrow G_{t}$ при $r \uparrow t$. Поэтому $\left(G_{t}\right)_{t \in \mathbf{R}}$ является правилом вхождения. 
Теперь мы укажем конструкцию процесса с иммиграцией, соответствуюшего $\left(N_{t}\right)_{t \geqslant 0}$. Следующая теорема показывает, что, за исключением детерминированной части $\left\{\gamma_{t}: t \geqslant 0\right\}$, и моменты вхождения, и эволюции иммигрантов определяются пуассоновской случайной мерой, основанной на мере Кузнецова $\mathbf{Q}^{G}$.

Теорема 4.1. Пусть $Y_{t}^{G}$ определяется соотношением (4.4) с $J=G, u$ пусть $Y_{t}=\gamma_{t}+Y_{t}^{G}$. Тогда $\left\{Y_{t}: t \geqslant 0\right\}-$ прочесс с иммиграчией с одномерными распределениями $\left(N_{t}\right)_{t \geqslant 0}$ и переходной полугрупnoŭ $\left(Q_{t}^{N}\right)_{t \geqslant 0}$.

Д о к а з а т е л ь с т в о. Предположим, что $\left(G_{t}\right)_{t \in \mathbf{R}}$ представляется в виде $(4.2)$ с $G_{s, t}$ вместо $J_{s, t}$. Тогда для $t \geqslant r \geqslant 0$

$$
\begin{aligned}
\int_{[r, t)} G_{s, t} \rho(d s) & =\int_{[0, t)} G_{s, t} \rho(d s)-\int_{[0, r)} G_{r, s} Q_{t-r}^{\circ} \rho(d s) \\
& =G_{t}-G_{r} Q_{t-r}^{\circ}=\int_{0}^{t} H_{s} d s-\int_{0}^{r} H_{s} Q_{t-r}^{\circ} d s .
\end{aligned}
$$

Из соотношения $K_{s+t}=K_{s} Q_{t}$ вытекает, что

$$
\eta_{s+t}=\int_{E} \eta_{s}(d x) \lambda_{t}(x, \cdot), \quad H_{s+t}=\int_{E} \eta_{s}(d x) L_{t}(x, \cdot)+H_{s} Q_{t}^{\circ}
$$

Из второго соотношения в (4.8) мы имеем

$$
\int_{0}^{r} H_{s+t-r} d s-\int_{0}^{r} H_{s} Q_{t-r}^{\circ} d s=\int_{0}^{r} d s \int_{E} \eta_{s}(d x) L_{t-r}(x, \cdot) .
$$

Подставляя это в (4.7), мы получаем

$$
\begin{aligned}
\int_{[r, t)} G_{t}^{s} \rho(d s) & =\int_{0}^{t-r} H_{s} d s+\int_{0}^{r} d s \int_{E} \eta_{s}(d x) L_{t-r}(x, \cdot) \\
& =G_{t-r}+\int_{E} \gamma_{r}(d x) L_{t-r}(x, \cdot) .
\end{aligned}
$$

Поскольку часть $\left\{\gamma_{t}: t \geqslant 0\right\}$ неслучайна, легко проверить, что $\left\{Y_{t}\right.$ : $t \geqslant 0\}-$ марковский процесс с одномерными распределениями $\left(N_{t}\right)_{t \geqslant 0}$. По лемме 4.1 мы имеем

$$
\begin{aligned}
\mathbf{E}\left[\exp \left\{-Y_{t}(f)\right\} \mid Y_{s}: 0 \leqslant s \leqslant r\right] \\
=\exp \left\{-Y_{r}^{G}\left(V_{t-r} f\right)-\gamma_{t}(f)\right. \\
\left.\quad-\int_{[r, t)} \rho(d s) \int_{M(E)^{\circ}}\left(1-e^{-\nu(f)}\right) G_{t}^{s}(d \nu)\right\} .
\end{aligned}
$$

Используя первое соотношение из (4.8), мы замечаем, что

$$
\begin{aligned}
\gamma_{t} & =\int_{0}^{t-r} \eta_{s} d s+\int_{0}^{r} d s \int_{E} \eta_{s}(d x) \lambda_{t-r}(x, \cdot) \\
& =\gamma_{t-r}+\int_{E} \gamma_{r}(d x) \lambda_{t-r}(x, \cdot)
\end{aligned}
$$


Комбинируя (4.9), (4.10) и (4.11), мы получаем

$$
\begin{aligned}
& \mathbf{E}\left[\exp \left\{-Y_{t}(f)\right\} \mid Y_{s}: 0 \leqslant s \leqslant r\right] \\
& \quad=\exp \left\{-Y_{r}\left(V_{t-r} f\right)-\gamma_{t-r}(f)-\int_{M(E)^{\circ}}\left(1-e^{-\nu(f)}\right) G_{t-r}(d \nu)\right\},
\end{aligned}
$$

т.е. $\left\{Y_{t}: t \geqslant 0\right\}$ - марковский процесс с переходной полугруппой $\left(Q_{t}^{N}\right)_{t \geqslant 0}$. Теорема доказана.

Теперь рассмотрим полугруппу $\left(Q_{t}\right)_{t \geqslant 0}(\xi, \phi)$-суперпроцесса. Пусть $\mathscr{K}^{1}(Q)$ - множество входных вероятностных законов $K=\left(K_{t}\right)_{t>0}$ для полугруппы $\left(Q_{t}\right)_{t \geqslant 0}$ таких, что

$$
\int_{0}^{1} d s \int_{M(E)^{\circ}} \nu(E) K_{s}(d \nu)<\infty .
$$

Пусть $\mathscr{K}(P)$ - множество входных законов $\kappa=\left(\kappa_{t}\right)_{t>0}$ для исходной полугруппы $\left(P_{t}\right)_{t \geqslant 0}$, которые удовлетворяют условию $\int_{0}^{1} \kappa_{s}(E) d s<\infty$. Для $\kappa \in \mathscr{K}(P)$ положим

$$
S_{t}(\kappa, f)=\kappa_{t}(f)-\int_{0}^{t} d s \int_{E} \phi\left(y, V_{s} f(y)\right) \kappa_{t-s}(d y)
$$

$t>0, f \in B(E)^{+}$. Заметим, что $S_{t}(\kappa, f)=\mu\left(V_{t} f\right)$, если $\left(\kappa_{t}\right)_{t>0}$ задается условием $\kappa_{t}=\mu P_{t}$. Следующая теорема полностью характеризует множество безгранично делимых входных вероятностных законов для $\left(Q_{t}\right)_{t \geqslant 0}$.

Теорема 4.2 (см. [26]). Захон $K \in \mathscr{K}^{1}(Q)$ безгранично делим тогда и только тогда, когда

$$
\ln L_{K_{t}}(f)=-S_{t}(\kappa, f)-\int_{\mathscr{K}(P)}\left(1-\exp \left\{-S_{t}(\eta, f)\right\}\right) F(d \eta),
$$

где $\kappa \in \mathscr{K}(P)$ и $F-\sigma$-конечная мера на $\mathscr{K}(P)$, удовлетворяючая условию

$$
\int_{0}^{1} d s \int_{\mathscr{K}(P)} \eta_{s}(1) F(d \eta)<\infty
$$

Пусть $\mathscr{K}\left(Q^{\circ}\right)$ - множество входных законов $K$ для $\left(Q_{t}^{\circ}\right)_{t \geqslant 0}$, удовлетворяющих (4.12). Для $\mathscr{K}\left(Q^{\circ}\right)$ можно также указать следующую общую характеризацию (см. также [7]).

Теорема 4.3. Любой входной захон $H \in \mathscr{K}\left(Q^{\circ}\right)$ может быть представлен в виде

$$
\begin{aligned}
& \int_{M(E)^{\circ}}\left(1-e^{-\nu(f)}\right) H_{t}(d \nu) \\
& \quad=S_{t}(\kappa, f)+\int_{\mathscr{K}(P)}\left(1-\exp \left\{-S_{t}(\eta, f)\right\}\right) F(d \eta)
\end{aligned}
$$


$t>0, f \in B(E)^{+}$, где $\kappa \in \mathscr{K}(P)$ и $F-\sigma$-конечная мера на $\mathscr{K}(P)$, удовлетворяющая (4.15). Если дополнительно

$$
\int_{a}^{\infty}\left[\sup _{x \in E}\left|\phi(x, z)^{-1}\right|\right] d z<\infty
$$

для некоторого постоянного а $>0$, то (4.16) определяет входной закон $H \in \mathscr{K}\left(Q^{\circ}\right)$ при любом $\kappa \in \mathscr{K}(P)$ и любой $\sigma$-конечной мере $F$ на $\mathscr{K}(P)$, удовлетворяющей (4.15).

Д о к а з а т е л ь с т в о. Если $H \in \mathscr{K}\left(Q^{\circ}\right)$, то $\left(K_{t}\right)_{t>0}=I\left(0, H_{t}\right)_{t>0}$ задает безгранично делимый входной вероятностный закон $K \in \mathscr{K}^{1}(Q)$. Таким образом, представление (4.16) вытекает из (4.14). Если выполнено (4.17), то существует семейство $\sigma$-конечных мер $\left\{L_{t}(x, \cdot): t>0\right.$, $x \in E\}$ на $M(E)^{\circ}$ таких, что $Q_{t}\left(\delta_{x}, \cdot\right)=I\left(0, L_{t}(x, \cdot)\right)$, см. $[4$, с. $195-$ 196]. Используя это, можно показать, что произвольный безгранично делимый входной вероятностный закон $K \in \mathscr{K}^{1}(Q)$ можно задать как $\left(K_{t}\right)_{t>0}=I\left(0, H_{t}\right)_{0}$ для некоторого $H \in \mathscr{K}\left(Q^{\circ}\right)$. Из (4.14) мы заключаем, что (4.16) определяет входной закон $H \in \mathscr{K}\left(Q^{\circ}\right)$. Теорема доказана.

Пусть $H \in \mathscr{K}\left(Q^{\circ}\right)$, и пусть $\mathbf{Q}^{H}-$ соответствующая мера Кузнецова с носителем $W_{0}(M(E))$. Если $N(d s, d w)$ - пуассоновская случайная мера на $[0, \infty) \times W_{0}(M(E))$ с интенсивностью $d s \times \mathbf{Q}^{H}(d w)$, то соотношение

$$
Y_{t}=\int_{[0, t)} \int_{W_{0}(M(E))} w_{t-s} N(d s, d w), \quad t \geqslant 0
$$

задает процесс с иммиграцией, соответствующий SC-полугруппе $\left(N_{t}\right)_{t \geqslant 0}$, определяемой с помошью соотношения

$$
\ln L_{N_{t}}(f)=-\int_{0}^{t} d s \int_{M(E)^{\circ}}\left(1-e^{-\nu(f)}\right) H_{s}(d \nu),
$$

$t \geqslant 0, f \in B(E)^{+}$. Ясно, что (4.18) по существу является частным случаем (4.4). Эта конструкция была использована в [26], [29] и [32]. Из теорем 3.2, 4.2 и 4.3 легко видеть, что при условии (4.17) любой однородный процесс с иммиграцией, ассоциированный с $(\xi, \phi)$-суперпроцессом, может быть задан в виде (4.18). С этим связана также статья [10], в которой условный $(\xi, \phi)$-суперпроцесс строится путем сложения масс, выкинутых «бессмертной частицей», двигающейся вокруг как копия $\xi$.

Конструкция, использующая процесс Кузнецова, позволяет обобщить некоторые известные результаты о $(\xi, \phi)$-суперпроцессах на процесс с иммиграцией. В качестве примера приведем характеризацию для «взвешенного времени занятости» процесса с иммиграцией с использованием конструкции (4.18). Для простоты мы рассмотрим только частный случай. Известно, что если $X-(\xi, \phi)$-суперпроцесс, то

$$
\mathbf{Q}_{\mu} \exp \left\{-X_{t}(f)-\int_{0}^{t} X_{s}(g) d s\right\}=\exp \left\{-\mu\left(V_{t}(f, g)\right)\right\}
$$


$f, g \in B(E)^{+}$, где $V_{t}(f, g)(x) \equiv u_{t}(x)-$ решение уравнения

$$
u_{t}(x)+\int_{0}^{t} d s \int_{E} \phi\left(x, u_{s}(y)\right) P_{t-s}(x, d y)=P_{t} f(x)+\int_{0}^{t} P_{s} g(x) d s
$$

$t \geqslant 0 ;$ см., например, [11] и [19]. Формулы (4.20) и (4.21) характеризуют совместное распределение $X_{t}$ и взвешенного времени занятости $\int_{0}^{t} X_{s} d s$. Из теорем 3.2 и 4.2 мы знаем, что условие

$$
\int_{M(E)} e^{-\nu(f)} Q_{t}^{\kappa}(\mu, d \nu)=\exp \left\{-\mu\left(V_{t} f\right)-\int_{0}^{t} S_{r}(\kappa, f) d r\right\}
$$

$t \geqslant 0, f \in B(E)^{+}$, определяет переходную полугруппу $\left(Q_{t}^{\kappa}\right)_{t \geqslant 0}$ процесса с иммиграцией, ассоциированного с $(\xi, \phi)$-суперпроцессом. Пусть

$$
h=\int_{0}^{1} P_{s} 1 d s \in B(E)^{+} .
$$

Из [26] мы знаем, что $\left(Q_{t}^{\kappa}\right)_{t \geqslant 0}$ имеет реализацию $\left(W, \mathscr{G}_{t}, \mathscr{G}_{t}, Y_{t}, \mathbf{Q}_{\mu}^{\kappa}\right)$ такую, что для каждого $g \in B(E)^{+}$траектория $\left\{Y_{t}(g \wedge h): t \geqslant 0\right\}$ почти наверное измерима и локально ограничена, откуда вытекает, что $\int_{0}^{t} Y_{s}(g) d s$ можно определить почти наверное с помощью возрастающих пределов.

Теорема 4.4. Предположим, ито выполнено условие (4.17). Пусть $\left(W, \mathscr{G}_{1}, \mathscr{G}_{t}, Y_{t}, \mathbf{Q}_{\mu}^{\kappa}\right)-$ реализачия $\left(Q_{t}^{\kappa}\right)_{t \geqslant 0}$, описанная выше. Тогда

$$
\begin{aligned}
& \mathbf{Q}_{\mu}^{\kappa} \exp \left\{-Y_{t}(f)-\int_{0}^{t} Y_{s}(g) d s\right\} \\
& \quad=\exp \left\{-\mu\left(u_{t}\right)-\int_{0}^{t} S_{r}(\kappa, f, g) d r\right\}, \quad f, g \in B(E)^{+},
\end{aligned}
$$

где $u_{t}(x)$ определяется из (4.21) $u$

$$
S_{t}(\kappa, f, g)=\kappa_{t}(f)+\int_{0}^{t} \kappa_{s}(g) d s-\int_{0}^{t} \kappa_{t-s}\left(\phi\left(u_{s}\right)\right) d s, \quad t>0 .
$$

Д о к а $з$ а т е л ь с т в о. По теореме 4.3 сушествует входной закон $H \in \mathscr{K}\left(Q^{\circ}\right)$ такой, что

$$
\int_{M(E)^{\circ}}\left(1-e^{-\nu(f)}\right) H_{t}(d \nu)=S_{t}(\kappa, f), \quad t>0, f \in B(E)^{+} .
$$

Пусть $\mathbf{Q}^{H}$ - соответствующая мера Кузнецова на $W(M(E))$. По (4.24) мы имеем

$$
\begin{aligned}
& \mathbf{Q}^{H}\left(1-\exp \left\{-w_{t}(f)-\int_{0}^{t} w_{s}(g) d s\right\}\right) \\
& \quad=\lim _{r \downarrow 0} \mathbf{Q}^{H}\left(1-\mathbf{Q}_{w_{r}} \exp \left\{-X_{t-r}(f)-\int_{0}^{t-r} X_{s}(g) d s\right\}\right) \\
& \quad=\lim _{r \downarrow 0} \mathbf{Q}^{H}\left(1-\exp \left\{-w_{r}\left(u_{t-r}\right)\right\}\right)=\lim _{r \downarrow 0} S_{r}\left(\kappa, u_{t-r}\right)=S_{t}(\kappa, f, g),
\end{aligned}
$$


где для получения последнего равенства использованы (4.21) и (4.23). Тогда, используя конструкцию (4.18), мы получаем

$$
\begin{aligned}
& \mathbf{Q}_{0}^{\kappa} \exp \left\{-Y_{t}(f)-\int_{0}^{t} Y_{s}(g) d s\right\} \\
& =\exp \left\{-\int_{0}^{t} \mathbf{Q}^{H}\left(1-\exp \left\{-w_{t-r}(f)-\int_{r}^{t} w_{s-r}(g) d s\right\}\right) d r\right\} \\
& =\exp \left\{-\int_{0}^{t} S_{t-r}(\kappa, f, g) d r\right\}
\end{aligned}
$$

и требуемый результат следует из соотношения $\mathbf{Q}_{\mu}^{\kappa}=\mathbf{Q}_{\mu} * \mathbf{Q}_{0}^{\kappa}$.

5. «Почти наверное»-поведение процессов Кузнецова. В этом пункте мы будем изучать поведение процессов Кузнецова в окрестностях моментов их рождения. Обсуждение этих свойств представляет интерес, поскольку оно проливает свет на структуру траекторий процесса с иммиграцией. Отсутствие феллеровского свойства снова делает доказательство немного длиннее, чем можно было бы ожидать. Пусть $\left(Q_{t}\right)_{t \geqslant 0}$ - переходная полугруппа $(\xi, \phi)$-суперпроцесса. Нам необходимо рассматривать две топологии на пространстве $E$ : исходную топологию и топологию Рэя процесса $\xi$. Мы используем обозначение $E_{r}$ для множества $E$ с топологией Рэя процесса $\xi$. Обозначение $M\left(E_{r}\right)$ самоочевидно. Пусть $\left(P_{t}^{b}\right)_{t \geqslant 0}$ - полугруппа ограниченных ядер на $E$, определяемых как

$$
P_{t}^{b} f(x)=\mathbf{P}_{x} f\left(\xi_{t}\right) \exp \left\{-\int_{0}^{t} b\left(\xi_{s}\right) d s\right\}, \quad x \in E, f \in B(E)^{+} .
$$

Несложно проверить, что для любого $H \in \mathscr{K}\left(Q^{\circ}\right)$ соотношение

$$
\gamma_{t}(f)=\int_{M(E)^{\circ}} \nu(f) H_{t}(d \nu), \quad t>0, f \in B(E)^{+}
$$

определяет входной закон $\gamma=\left(\gamma_{t}\right)_{t>0}$ для $\left(P_{t}^{b}\right)_{t \geqslant 0}$.

Сначала рассмотрим специальный $\sigma$-конечный входной закон. Вспомним общую формулу (4.16). Пусть $x \in E$. Предположим, что соотношение

$$
\int_{M(E)^{\circ}}\left(1-e^{-\nu(f)}\right) L_{t}(x, d \nu)=V_{t} f(x), \quad t>0, f \in B(E)^{+},
$$

определяет входной закон $L(x) \in \mathscr{K}\left(Q^{\circ}\right)$. Ясно, что $\left(P_{t}^{b}(x, \cdot)\right)_{t>0}$ - минимальный входной закон для $\left(P_{t}^{b}\right)_{t \geqslant 0}$, который может быть задан соотношением (5.2) с $H_{t}(d \nu)$, замененным на $L_{t}(x, d \nu)$. Отсюда легко вывести, что $L(x) \in \mathscr{K}\left(Q^{\circ}\right)$ минимален.

Теорема 5.1. Обозначим $\mathbf{Q}^{L(x)}$ меру Кузнечова на $W(M(E))$, определяемую соотношением $L(x) \in \mathscr{K}\left(Q^{\circ}\right)$. Тогда $w_{t}(E) \rightarrow 0$ $u\left(w_{t}(E)\right)^{-1} w_{t} \rightarrow \delta_{x}$ в $M\left(E_{r}\right)$ nрu $t \downarrow 0$ для $\mathbf{Q}^{L(x)}$-почти всех траекторий $w \in W(M(E))$. 
Д о к а з а т е л с с т в о. Утверждение было доказано в [29] для случая, когда полугруппа $\left(P_{t}\right)_{t \geqslant 0}$ является феллеровской и $\phi(x, z) \equiv z^{2} / 2$ по теореме Перкинса [30], которая утверждает, что условный $(\xi, \phi)$-суперпроцесс является обобщенным суперпроцессом Флеминга-Вио. Выкладки в [29] громоздки и не могут быть обобщены на рассматриваемую ситуацию. Здесь мы приведем доказательство теоремы, основанное на $h$-преобразовании $(\xi, \phi)$-суперпроцесса. В нашем доказательстве важную роль играет рэевский конус для исходного процесса $\xi$. Мы будем предполагать, что полугруппа $\left(P_{t}\right)_{t \geqslant 0}$ консервативна. Для неконсервативной исходной полугруппы доказательство может быть сведено к консервативному случаю подобно тому, как это сделано в [29].

Пусть $\mathscr{R}$ - счетный конус Рэя для $\xi$, построенный в [31], и пусть $\bar{E}$ - соответствующая компактификация Рэя-Найта множества $E$ с топологией Рэя. Заметим, что каждая $f \in \mathscr{R}$ непрерывна на $E_{r}$ и допускает непрерывное продолжение $\bar{f}$ на $\bar{E}$. Как обычно, мы рассматриваем $M\left(E_{r}\right)$ как топологическое подпространство $M(\bar{E})$. Поскольку $\bar{E}$ является компактным метрическим пространством, $M(\bar{E})$ локально компактно и сепарабельно. Для любого фиксированного $u>0$ соотношение

$$
U_{t}^{r}(\mu, d \nu)=\left(\mu\left(P_{u-r}^{b} 1\right)\right)^{-1} \nu\left(P_{u-t}^{b} 1\right) Q_{t-r}(\mu, d \nu), \quad 0 \leqslant r \leqslant t \leqslant u,
$$

определяет неоднородную переходную полугруппу $\left(U_{t}^{r}\right)_{r \leqslant t}$ на $M(E)^{\circ}$. Определим вероятностную меру $\mathbf{U}_{u}^{L(x)}(d w)$ на $W(M(E))$ с помощью соотношения

$$
\mathbf{U}_{u}^{L(x)}(d w)=\left(P_{u}^{b} 1(x)\right)^{-1} w_{u}(1) \mathbf{Q}^{L(x)}(d w) .
$$

Тогда относительно $\mathbf{U}_{u}^{L(x)}$ процесс $\left\{w_{t}: 0<t \leqslant u\right\}$ является марковским с полугруппой $\left(U_{t}^{r}\right)_{r \leqslant t}$ и одномерными распределениями

$$
H_{t}(x, d \nu):=\left(P_{u}^{b} 1(x)\right)^{-1} \nu\left(P_{u-t}^{b} 1\right) L_{t}(x, d \nu), \quad 0<t \leqslant u .
$$

Поскольку $L(x) \in \mathscr{K}\left(Q^{\circ}\right)$ минимален, $\left(H_{t}(x, \cdot)\right)_{0<t \leqslant u}$ является минимальным входным (вероятностным) законом для $\left(U_{t}^{r}\right)_{r \leqslant t}$. Возьмем $f \in \mathscr{R}$. По $(5.3)-(5.5)$ и теореме о мартингальной сходимости мы $\mathrm{U}_{u}^{L(x)}$-почти наверное имеем

$$
\begin{aligned}
V_{t} f(x) & =\int_{M(E)^{\circ}}\left(1-e^{-\nu(f)}\right)\left(\nu\left(P_{u-t}^{b} 1\right)\right)^{-1} H_{t}(x, d \nu) P_{u}^{b} 1(x) \\
& =\lim _{r \downarrow 0} \int_{M(E)^{\circ}}\left(1-e^{-\nu(f)}\right)\left(\nu\left(P_{u-t}^{b} 1\right)\right)^{-1} U_{t}^{r}\left(w_{r}, d \nu\right) P_{u}^{b} 1(x) \\
& =\lim _{r \downarrow 0}\left(w_{r}\left(P_{u-r}^{b} 1\right)\right)^{-1}\left(1-\exp \left\{-w_{r}\left(V_{t-r} f\right)\right\}\right) P_{u}^{b} 1(x) .
\end{aligned}
$$

Из (5.1) и (5.6) вытекает, что $\mathrm{U}_{u}^{L(x)}$-почти наверное

$$
V_{t} f(x) \leqslant \liminf _{r \downarrow 0}\left(w_{r}\left(P_{u-r}^{b} 1\right)\right)^{-1} P_{u}^{b} 1(x) \leqslant \liminf _{r \downarrow 0} e^{2\|b\| u}\left(w_{r}(1)\right)^{-1} .
$$


Заметим, что $V_{t} f(x)$ непрерывна справа по $t \geqslant 0$. Тогда, устремляя $t \downarrow 0$ и $f \uparrow \infty$ в последнем неравенстве, мы получаем, что $\mathbf{U}_{u}^{L(x)}$-почти наверное $w_{t}(1) \rightarrow 0$ при $t \downarrow 0$. Поскольку при каждом $u>0$ меры $\mathbf{U}_{u}^{L(x)}$ и $\mathbf{Q}^{L(x)}$ взаимно абсолютно непрерывны на $\left\{w \in W_{0}(M(E)): w_{u}(1)>0\right\}$, мы получаем первое утверждение. Рассуждая так же, как в (5.6), мы получаем, что $\mathrm{U}_{t}^{L(x)}$-почти наверное

$$
\begin{aligned}
P_{t}^{b} f(x) & =\int_{M(E)^{\circ}} \nu(f)(\nu(1))^{-1} H_{t}(x, d \nu) P_{t}^{b} 1(x) \\
& =\lim _{r \downarrow 0}\left(w_{r}\left(P_{t-r}^{b} 1\right)\right)^{-1} w_{r}\left(P_{t-r}^{b} f\right) P_{t}^{b} 1(x) .
\end{aligned}
$$

Понятно, что мера $\mathbf{U}_{u}^{L(x)}$ абсолютно непрерывна относительно $\mathbf{U}_{t}^{L(x)}$ при $u \geqslant t>0$. Так как $f \in \mathscr{R}$ является $\alpha$-избыточной функцией для $\left(P_{t}\right)_{t \geqslant 0}$ при некотором $\alpha=\alpha(f) \geqslant 0$, то из (5.1) и (5.7) вытекает, что $\mathbf{U}_{u}^{L(x)}$-почти наверное

$$
e^{-\|b\| t} P_{t} f(x) \leqslant \liminf _{r \downarrow 0} e^{(3\|b\|+\alpha) t}\left(w_{r}(1)\right)^{-1} w_{r}(f) .
$$

Возьмем $w \in W_{0}(M(E))$, вдоль которой выполняется последнее неравенство для всех $f \in \mathscr{R}$ и всех рациональных $t \in(0, u]$. Пусть $r_{k}=r_{k}(w)-$ последовательность такая, что $r_{k} \downarrow 0$ и $\left(w_{r_{k}}(1)\right)^{-1} w_{r_{k}} \rightarrow \widehat{w}_{0}$ в $M(\bar{E})$ при $k \rightarrow \infty$, где $\widehat{w}_{0}-$ вероятностная мера на $\bar{E}$. Тогда

$$
e^{-\|b\| t} P_{t} f(x) \leqslant e^{(3\|b\|+\alpha) t} \widehat{w}_{0}(\bar{f}) .
$$

Устремив $t \downarrow 0$, мы получим $f(x) \leqslant \widehat{w}_{0}(\bar{f})$, так что $\widehat{w}_{0}=\delta_{x}$. Отсюда с очевидностью следует, что $\left(w_{r}(1)\right)^{-1} w_{r} \rightarrow \delta_{x}$ в $M\left(E_{r}\right)$ при $r \downarrow 0$, откуда непосредственно вытекает второе утверждение.

Теперь мы рассмотрим $h$-преобразование исходной полугруппы $\left(P_{t}\right)_{t \geqslant 0}$. Пусть $h(x)=\int_{0}^{1} P_{s} 1(x) d s$ для $x \in E$. Так как $h \in B(E)^{+}-$ избыточная функция для $\left(P_{t}\right)_{t \geqslant 0}$, то формула

$$
T_{t} f(x)=(h(x))^{-1} \int_{E} f(y) h(y) P_{t}(x, d y), \quad x \in E, f \in B(E)^{+},
$$

определяет правую борелевскую полугруппу $\left(T_{t}\right)_{t \geqslant 0}$ на $E$, см., например, [31]. Пусть $\left(T_{t}^{\partial}\right)_{t \geqslant 0}-$ консервативное продолжение $\left(T_{t}\right)_{t \geqslant 0}$ на $E^{\partial}:=E \cup\{\partial\}$, где $\partial-$ точка покоя. Пусть $E_{D}^{\partial}$ обозначает входное пространство $\left(T_{t}^{\partial}\right)_{t \geqslant 0}$ с топологией Рэя. Пусть $E_{D}^{T}=E_{D}^{\partial} \backslash\{\partial\}$, и пусть $\left(\bar{T}_{t}\right)_{t \geqslant 0}-$ рэевское продолжение $\left(T_{t}^{\partial}\right)_{t \geqslant 0}$ на $E_{D}^{T}$. Тогда $\left(\bar{T}_{t}\right)_{t \geqslant 0}$ также являются правыми борелевскими полугруппами. Пусть закон $\kappa \in \mathscr{K}(P)$, нетривиален, и предположим, что соотношение

$$
\int_{M(E)^{\circ}}\left(1-e^{-\nu(f)}\right) H_{t}(d \nu)=S_{t}(\kappa, f), \quad t>0, f \in B(E)^{+},
$$

задает входной закон $H:=L \kappa \in \mathscr{K}\left(Q^{\circ}\right)$. Пусть $\mathbf{Q}^{L \kappa}-$ соответствующая мера Кузнецова на $W(M(E))$. Тогда справедлива 
Теорема 5.2. Для $w \in W(M(E))$ определим $M\left(E_{D}^{T}\right)$-значную траекторию $\left\{h \bar{w}_{t}: t \in \mathbf{R}\right\}$ с помощью соотношений

$$
h \bar{w}_{t}\left(E_{D}^{T} \backslash E\right)=0 \quad u \quad h \bar{w}_{t}(d x)=h(x) w_{t}(d x) \quad \partial \Omega \Omega x \in E .
$$

Tогда для $\mathbf{Q}^{L \kappa}$-nочти всех $w \in W(M(E))$ nрочесc $\left\{h \bar{w}_{t}: t>0\right\}$ непрерывен справа в топологии $M\left(E_{D}^{T}\right)$ u $h \bar{w}_{t} \rightarrow 0$ при $t \downarrow 0$. При этом $\partial \Omega s$ $\mathbf{Q}^{L \kappa}$-почти всех $w \in W(M(E))$ мы имеем $\left(w_{t}(h)\right)^{-1} h \bar{w}_{t} \rightarrow \delta_{x(w)}$ для некоторого $x(w) \in E_{D}^{T}$ npu $t \downarrow 0$.

Д ок аз а т е ль с т в о. В силу результатов [11], если $f \in B(E)$ чисто непрерывна относительно $\left(P_{t}\right)_{t \geqslant 0}$, то $\left\{w_{t}(f): t>0\right\}$ непрерывна справа для почти всех $w \in W(M(E))$. Так как избыточная функция $h \in B(E)^{+}$чисто непрерывна, то этим свойством обладает и $f h$, какова бы ни была ограниченная непрерывная функция $f$ на $E$. Следовательно, $\left\{h w_{t}: t>0\right\}$ непрерывен справа для почти всех $w \in W(M(E))$. Мы можем определить полугруппу кумулянтов $\left(U_{t}\right)_{t \geqslant 0}$ как $U_{t} f=h^{-1} V_{t}(h f)$. Тогда $\left\{h w_{t}: t>0\right\}$ - марковский процесс с правой борелевской переходной полугруппой, заданной соотношением $(1.3)$, в котором $\left(V_{t}\right)_{t \geqslant 0}$ заменено на $\left(U_{t}\right)_{t \geqslant 0}$. Пусть $E_{r}^{T}$ обозначает множество $E$, снабженное относительной топологией из множества $E_{D}^{T}$. Снова применяя результаты из [11], мы заключаем, что $\left\{h w_{t}: t>0\right\}$ непрерывен справа в $M\left(E_{r}^{T}\right)$ для почти всех $w \in W(M(E))$. Поэтому $\left\{h \bar{w}_{t}: t>0\right\}$ непрерывен справа в $M\left(E_{D}^{T}\right)$ для почти всех $w \in W(M(E))$. Заметим, что соотношение

$$
\bar{\psi}(x, z)= \begin{cases}(h(x))^{-1} \phi(x, h(x) z), & \text { если } x \in E, \\ 0, & \text { если } x \in E_{D}^{T} \backslash E,\end{cases}
$$

определяет механизм ветвления $\bar{\psi}(\cdot, \cdot)$ на $E_{D}^{T}$. Пусть $\left(\bar{U}_{t}\right)_{t \geqslant 0}-$ полугруппа кумулянтов, определяемая соотношением

$$
\bar{U}_{t} \bar{f}(x)+\int_{0}^{t} d s \int_{E_{D}^{T}} \bar{\psi}\left(y, \bar{U}_{s} \bar{f}(y)\right) \bar{T}_{t-s}(x, d y)=\bar{T}_{t} \bar{f}(x),
$$

$t \geqslant 0, x \in E_{D}^{T}$. Тогда $\left(\bar{U}_{t}\right)_{t \geqslant 0}$ соответствует борелевской правой переходной полугруппе $\left(\bar{Q}_{t}\right)_{t \geqslant 0}$ на $M\left(E_{D}^{T}\right)$. Для любых $t>0$ и $x \in E_{D}^{T}$ носителем меры $\bar{T}_{t}(x, \cdot)$ является $E$, так что $\bar{T}_{t} \bar{f}(x)$ и $\bar{U}_{t} \bar{f}(x)$ не зависят от значений $\bar{f}$ на $E_{D}^{T} \backslash E$. В самом деле, если $f=\left.\bar{f}\right|_{E}$ для $f \in B\left(E_{D}^{T}\right)^{+}$, то $\bar{U}_{t} \bar{f}(x)=U_{t} f(x)$ для всех $x \in E$. Мы можем написать $\bar{T}_{t} f$ и $\bar{U}_{t} f$ вместо $\bar{U}_{t} \bar{f}$ и $\bar{U}_{t} \bar{f}$ соответственно. Ясно, что определение $\bar{T}_{t} f$ и $\bar{U}_{t} f$ можно распространить на все неотрицательные борелевские функции $f$ на $E$ с помощью возрастающих пределов. Как показано в [26], существует мера $\rho \in M\left(E_{D}^{T}\right)$ такая, что $\kappa_{t}(f)=\rho\left(\bar{T}_{t}\left(h^{-1} f\right)\right)$ и $S_{t}(\kappa, f)=\rho\left(\bar{U}_{t}\left(h^{-1} f\right)\right)$. Тогда $\left\{h \bar{w}_{t}: t>0\right\}-$ марковский процесс с переходной полугруппой $\left(\bar{Q}_{t}\right)_{t \geqslant 0}$ и

$$
\mathbf{Q}^{L \kappa}\left(1-e^{-h \bar{w}_{t}(\bar{f})}\right)=\rho\left(\bar{U}_{t} \bar{f}\right), \quad t>0, f \in B\left(E_{D}^{T}\right)^{+} .
$$


Теперь требуемые результаты вытекают из теоремы 5.1, примененной $\mathbf{~ к ~}\left(\bar{U}_{t}\right)_{t \geqslant 0}$ и $\left(\bar{T}_{t}\right)_{t \geqslant 0}$.

В силу (4.14) мы имеем входной закон $K:=l \kappa \in \mathscr{K}^{1}(Q)$, задаваемый соотношением

$$
\int_{M(E)} e^{-\nu(f)} K_{t}(d \nu)=\exp \left\{-S_{t}(\kappa, f)\right\}, \quad t>0, f \in B(E)^{+} .
$$

Легко видеть, что сужение $K$ на $M(E)^{\circ}$ принадлежит к $\mathscr{K}\left(Q^{\circ}\right)$. Пусть $\mathbf{Q}^{l \kappa}$ обозначает соответствующую меру Кузнецова на $W(M(E))$.

Теорема 5.3. Для $\mathbf{Q}^{l \kappa}-$ почти всех $w \in W(M(E))\left\{h \bar{w}_{t}: t>0\right\}$ непрерывен справа $и h \bar{w}_{t} \rightarrow \rho$ для нехоторого $\rho \in M\left(E_{D}^{T}\right) n p u t \downarrow 0$.

Д ок а з а т ль с т о. Мы используем обозначения, введенные при доказательстве теоремы 5.2. Ясно, что $\left\{h \bar{w}_{t}: t>0\right\}$ является марковским процессом относительно $\mathbf{Q}^{l \kappa}$ с переходной полугруппой $\left(\bar{Q}_{t}\right)_{t \geqslant 0}$ и

$$
\mathbf{Q}^{l \kappa} \exp \left\{-h \bar{w}_{t}(\bar{f})\right\}=\exp \left\{-\rho\left(\bar{U}_{t} \bar{f}\right)\right\}, \quad t>0, f \in B\left(E_{D}^{T}\right)^{+} .
$$

Таким образом, требуемые утверждения справедливы в силу единственности переходной вероятности.

Наконец, рассмотрим поведение траекторий процесса Кузнецова, определенного с помощью общего правила вхождения. Пусть $\left(J_{t}\right)_{t \in \mathbf{R}}-$ правило вхождения для $\left(Q_{t}^{\circ}\right)_{t \geqslant 0}$, удовлетворяющее условию

$$
\int_{r}^{t} d s \int_{M(E)^{\circ}} \nu(E) J_{s}(d \nu)<\infty, \quad r \leqslant t, r, t \in \mathbf{R} .
$$

Тогда мы можем считать, что $\left(J_{t}\right)_{t \in \mathbf{R}}$ задано соотношением (4.2) с входными законами $\left\{\left(H_{s, s+t}\right)_{t>0}: s \in \mathbf{R}\right\}$, взятыми из $\mathscr{K}\left(Q^{\circ}\right)$.

Теорема 5.4. $B$ описанной выше ситуачии для $\mathbf{Q}^{J}$-почти всех траекторий $w \in W(M(E))$ прочесс $\left\{h \bar{w}_{t}: t \in \mathbf{R}\right\}$, определенный в (5.10), непрерывен справа в $M\left(E_{D}^{T}\right)^{\circ}$ на интервале $(\alpha(w), \beta(w)) u h \bar{w}_{t} \rightarrow h \bar{w}_{\alpha}$ для нехоторого $h \bar{w}_{\alpha} \in M\left(E_{D}^{T}\right) n p u t \downarrow \alpha(w)$. При этом для $\mathbf{Q}^{J}$-почти всех траекторий $w \in W(M(E))$ с $h \bar{w}_{\alpha}=0$ мы имеем $\left(w_{t}(h)\right)^{-1} h \bar{w}_{t} \rightarrow$ $\delta_{x(w)}$ для некоторого $x(w) \in E_{D}^{T}$ nрu $t \downarrow \alpha(w)$.

Д ок а з а т е л с т в о. Пусть $\mathbf{Q}^{H}$ - мера Кузнецова на $W(M(E))$, соответствуюшая входному закону $H \in \mathscr{K}\left(Q^{\circ}\right)$, представленному соотношением (4.16). Тогда мы имеем

$$
\mathbf{Q}^{H}(d w)=\mathbf{Q}^{L \kappa}(d w)+\int_{\mathscr{K}(P)} \mathbf{Q}^{l \eta}(d w) F(d \eta), \quad w \in W(M(E)) .
$$

По теоремам 5.2 и 5.3 для $\mathbf{Q}^{H}$-почти всех $w \in W(M(E))$ процесс $\left\{h \bar{w}_{t}: t>0\right\}$ непрерывен справа в $M\left(E_{D}^{T}\right)$ и $h \bar{w}_{t} \rightarrow h \bar{w}_{0}$ для некоторого $h \bar{w}_{0} \in M\left(E_{D}^{T}\right)$ при $t \downarrow 0$. Более того, для $\mathbf{Q}^{H}$-почти всех $w \in W(M(E))$ с $h \bar{w}_{0}=0$ мы имеем $\left(w_{t}(h)\right)^{-1} h \bar{w}_{t} \rightarrow \delta_{x(w)}$ для некоторого $x(w) \in E_{D}^{T}$ при $t \downarrow 0$. Тогда требуемый результат имеет место в силу представления (4.3) меры $\mathbf{Q}^{J}(d w)$. 
Ясно, что соотношению (5.13) удовлетворяет правило вхождения $\left(G_{t}\right)_{t \in \mathbf{R}}$ в теореме 4.1. Следуюший пример показывает, что рассматривать процесс $\left\{h \bar{w}_{t}: t \geqslant 0\right\}$ необходимо, если требуется получить правый предел траектории в $\alpha=\alpha(w)$ в обычном смысле.

П р и м е р 5.1. Предположим, что $\xi-$ минимальное броуновское движение в ограниченной области $D \subset \mathbf{R}^{d}$ с гладкой границей $\partial D$. Мы также используем символ $\partial$ для обозначения оператора внутренней нормальной производной на $\partial D$. Для любого $\gamma \in M(\partial D)$ определим $\left(G_{t}\right)_{t>0}$ соотношением

$\int_{M(D)^{\circ}}\left(1-e^{-\nu(f)}\right) G_{t}(d \nu)=\int_{0}^{t}\left(1-\exp \left\{-\gamma\left(\partial V_{t-s} f\right)\right\}\right) d s, \quad f \in B(D)^{+}$,

и положим $G_{t}=0$ при $t \leqslant 0$. Тогда $\left(G_{t}\right)_{t \in \mathbf{R}}$ образует правило вхождения для $(\xi, \phi)$-суперпроцесса. $\mathrm{C}$ помощью простых модификаций доказательств теорем 5.3 и 5.4 можно убедиться, что $w_{\alpha+}(D)=\infty$ и $w_{\alpha+}(K)=0$ для всех компактных множеств $K \subset D$ и $\mathbf{Q}^{G}$-почти всех траекторий $w \in W(M(D))$.

6. Стационарные процессы с иммиграцией. Процессы с иммиграцией, описываемые с помощью SC-полугрупп, тесно связаны с теорией избыточных мер, см., например, [13], [14] и [6]. В этом пункте мы сформулируем некоторые результаты об избыточных мерах в терминах стационарных процессов с иммиграцией.

Сначала рассмотрим полугруппу $\left(Q_{t}\right)_{t \geqslant 0}$ общего правого борелевского MB-процесса. Для двух вероятностных мер $F_{1}$ и $F_{2}$ на $M(E)$ мы будем писать $F_{1} \preceq F_{2}$, если существует некоторая вероятностная мера $G$ такая, что $F_{1} * G=F_{2}$. Пусть $\mathscr{E}^{*}(Q)$ обозначает множество всех вероятностных мер $F$ на $M(E)$ таких, что

$$
\int_{M(E)^{\circ}} \nu(1) F(d \nu)<\infty
$$

и $F Q_{t} \preceq F$ для всех $t \geqslant 0$. Мы будем писать $F \in \mathscr{E}_{i}^{*}(Q)$, если $F \in$ $\mathscr{E}^{*}(Q)$ - стационарное распределение для $\left(Q_{t}\right)_{t \geqslant 0}$, и $F \in \mathscr{E}_{p}^{*}(Q)$, если $F \in \mathscr{E}^{*}(Q)$ и $\lim _{t \rightarrow \infty} F Q_{t}=\delta_{0}$. Очевидно, что $\delta_{0} \in \mathscr{E}_{i}^{*}(Q)$, но могут существовать другие нетривиальные стационарные распределения, хотя мы и рассматриваем фазовое пространство $M(E)$.

Пусть $\mathscr{E}\left(Q^{\circ}\right)$ обозначает класс всех избыточных мер $F$ для $\left(Q_{t}^{\circ}\right)_{t \geqslant 0}$, удовлетворяющих (6.1). Пусть $\mathscr{E}_{i}\left(Q^{\circ}\right)-$ подмножество множества $\mathscr{E}\left(Q^{\circ}\right)$, включающее в себя все инвариантные меры, и пусть $\mathscr{E}_{p}\left(Q^{\circ}\right)-$ подмножество чисто избыточных мер. Классы $\mathscr{E}\left(Q^{\circ}\right)$ и $\mathscr{E}^{*}(Q)$ тесно связаны. В самом деле, мера $F \in \mathscr{E}^{*}(Q)$ безгранично делима тогда и только тогда, когда $F=I(\rho, J)$ для $\rho \in M(E)$ и $J \in \mathscr{E}\left(Q^{\circ}\right)$, удовлетворяюшего условиям

$$
\int_{E} \rho(d x) \lambda_{t}(x, \cdot) \leqslant \rho \quad \text { и } \int_{E} \rho(d x) L_{t}(x, \cdot)+J Q_{t}^{\circ} \leqslant J .
$$


При условии (4.17) мера $F \in \mathscr{E}^{*}(Q)$ безгранично делима тогда и только тогда, когда $F=I(0, J)$ для некоторого $J \in \mathscr{E}\left(Q^{\circ}\right)$.

Следующая теорема показывает, что $\mathscr{E}^{*}(Q)$ совпадает с множеством стационарных распределений процессов с иммиграцией, связанных с $X$.

Теорема 6.1. Пусть $F \in \mathscr{E}^{*}(Q)$. Тогда эта мера может быть единственным образом представлена в виде $F=F_{i} * F_{p}$, где $F_{i}=$ $\lim _{t \rightarrow \infty} F Q_{t} \in \mathscr{E}_{i}^{*}(Q)$ u $F_{p} \in \mathscr{E}_{p}^{*}(Q)$. При этом существует единственная $S C$-полугруппа $\left(N_{t}\right)_{t \geqslant 0}$ такая, что $\lim _{t \rightarrow \infty} N_{t}=F_{p}$.

Д ок а з а т е л ь с т о. Пусть $\left(N_{t}\right)_{t \geqslant 0}$ - распределения на $M(E)$, удовлетворяющие условию $F=\left(F Q_{t}\right) * N_{t}$. В силу свойства ветвления полугруппы $\left(Q_{t}\right)_{t \geqslant 0}$ можно проверить, что для любых $r \geqslant 0$ и $t \geqslant 0$

$$
\begin{aligned}
\left(F Q_{r+t}\right) * N_{r+t} & =F=\left(F Q_{t}\right) * N_{t}=\left\{\left[\left(F Q_{r}\right) * N_{r}\right] Q_{t}\right\} * N_{t} \\
& =\left(F Q_{r+t}\right) *\left(N_{r} Q_{t}\right) * N_{t} .
\end{aligned}
$$

Следовательно, семейство $\left(N_{t}\right)_{t \geqslant 0}$ удовлетворяет соотношению (1.4), так что оно является SC-полугруппой, ассоциированной с $\left(Q_{t}\right)_{t \geqslant 0}$. По определению $\mathscr{E}^{*}(Q)$ мы имеем $F Q_{r+t} \preceq F Q_{t}$, так что существуют следующие пределы, определяющие функционалы Лапласа двух вероятностных мер $F_{i}$ и $F_{p}$ :

$$
L_{F_{i}}(f)=\uparrow \lim _{t \uparrow \infty} L_{F Q_{t}}(f), \quad L_{F_{p}}(f)=\downarrow \lim _{t \uparrow \infty} L_{N_{t}}(f), \quad f \in B(E)^{+} .
$$

Очевидно, что $F_{i} \in \mathscr{E}_{i}^{*}(Q)$ и $F=F_{i} * F_{p}$. С другой стороны, $F_{i} * F_{p}=$ $F=\left(F Q_{t}\right) * N_{t}=F_{i} *\left(F_{p} Q_{t}\right) * N_{t}$, так что $F_{p}=\left(F_{p} Q_{t}\right) * N_{t}$. Поэтому $F_{p} \in \mathscr{E}^{*}(Q)$ и $\lim _{t \rightarrow \infty} F_{p} Q_{t}=\delta_{0}$. Единственность разложения очевидна.

Хорошо известно, что любое $J \in \mathscr{E}\left(Q^{\circ}\right)$ имеет разложение Рисса $J=J_{i}+J_{p}$, где $J_{i} \in \mathscr{E}_{i}\left(Q^{\circ}\right)$ и $J_{p} \in \mathscr{E}_{p}\left(Q^{\circ}\right)$ можно представить в виде $J_{p}=\int_{0}^{\infty} H_{t} d t$ для некоторого $H \in \mathscr{K}\left(Q^{\circ}\right)$. Пусть $\mathbf{Q}^{J}-$ мера Кузнецова на $W(M(E))$, определяемая $J$, и пусть $N^{J}(d w)$ - пуассоновская случайная мера с интенсивностью $\mathbf{Q}^{J}(d w)$. По лемме 4.1 соотношение

$$
Y_{t}^{J}:=\int_{W(M(E))} w_{t} N^{J}(d w), \quad t \in \mathbf{R},
$$

определяет стационарный процесс с иммиграцией с одномерным распределением $I(0, J)$, соответствующий SC-полугруппе $\left(N_{t}\right)_{t \geqslant 0}$, определенной соотношением (4.19). Меры Кузнецова, определяемые $J_{i}$ и $J_{p}$, являются сужениями $\mathbf{Q}^{J}$ на $\{w \in W(M(E)): \alpha(w)=-\infty\}$ и $\{w \in$ $W(M(E)): \alpha(w)>-\infty\}$ соответственно; см. [13]. Следовательно, соотношение

$$
Y_{t}^{(p)}=\int_{W(M(E))} w_{t} 1_{\{\alpha>-\infty\}} N^{J}(d w), \quad t \in \mathbf{R},
$$

определяет стационарный процесс с иммиграцией, имеющий одномерное распределение $I\left(0, J_{p}\right)$ и $\mathrm{SC}$-полугруппу $\left(N_{t}\right)_{t \geqslant 0}$. Интуитивно ясно, 
что $\left\{Y_{t}^{(p)}: t \in \mathbf{R}\right\}$ является «чисто иммиграционной» частью популяции. Напротив,

$$
Y_{t}^{(i)}=\int_{W(M(E))} w_{t} 1_{\{\alpha=-\infty\}} N^{J}(d w), \quad t \in \mathbf{R},
$$

является стационарным $(\xi, \phi)$-суперпроцессом с одномерным распределением $I\left(0, J_{i}\right)$, который представляет «коренную» часть популяции.

Процесс с иммиграцией, определяемый соотношением (6.5), как правило, не является непрерывным справа, но может иметь непрерывную справа модификацию. Для $w \in W(M(E))$ положим

$$
w_{t+}= \begin{cases}\lim _{s \downarrow t} w_{s}, & \text { если предел в } M(E) \text { существует, } \\ 0, & \text { если указанный выше предел в } M(E) \\ & \text { не существует }\end{cases}
$$

и определим процесс $\left\{\bar{Y}_{t}^{J}: t \in \mathbf{R}\right\}$ как

$$
\bar{Y}_{t}^{J}=\int_{W(M(E))} w_{t+} N^{J}(d w), \quad t \in \mathbf{R} .
$$

Тогда $\bar{Y}_{t}^{J}=Y_{t}^{J}$ почти наверное, так как $\mathbf{Q}^{J}\left\{w_{t+} \neq w_{t}\right\}=\mathbf{Q}^{J}\{\alpha=t\}=0$, см. [13]. Другими словами, $\left\{\bar{Y}_{t}^{J}: t \in \mathbf{R}\right\}$ является модификацией $\left\{Y_{t}^{J}\right.$ : $t \in \mathbf{R}\}$.

Теорема 6.2. Предположим, ито $\left(Q_{t}\right)_{t \geqslant 0}-$ переходная полугрупna $(\xi, \phi)$-суперпрочесса. (i) Ecлu $J \in \mathscr{E}_{i}\left(Q^{\circ}\right)$, mo прочесc $\left\{\bar{Y}_{t}^{J} \equiv Y_{t}^{J}\right.$ : $t \in \mathbf{R}\}$ почти наверное непрерывен справа. (ii) Eсли $J \in \mathscr{E}_{p}\left(Q^{\circ}\right)$ потенчиал меры, т.е.

$$
\int_{M(E)^{\circ}}\left(1-e^{-\nu(f)}\right) J(d \nu)=\int_{0}^{\infty} d s \int_{M(E)^{\circ}}\left(1-\exp \left\{-\nu\left(V_{s} f\right)\right\}\right) G(d \nu)
$$

$f \in B(E)^{+}$, где $\nu(1) G(d \nu)$ - конечная мера на $M(E)^{\circ}$, то прочесс $\left\{\bar{Y}_{t}^{J}: t \in \mathbf{R}\right\}$ почти наверное непрерывен справа.

Д ок а з т е л ь т в о. Поскольку утверждение (i) весьма просто, мы приведем лишь доказательство утверждения (ii). Для $k=1,2, \ldots$ положим

$$
W_{k}(M(E))=\left\{w \in W(M(E)): w_{\alpha+}(E) \geqslant \frac{1}{k}\right\} .
$$

Согласно результатам [13], траектория $\left\{w_{t+}: t \in \mathbf{R}\right\}$ непрерывна справа для $\mathbf{Q}^{J}$-почти всех $w \in W(M(E))$ и $\mathbf{Q}^{J}\left(\left[\cup_{k=1}^{\infty} W_{k}(M(E))\right]^{c}\right)=0$. Пусть

$$
\bar{Y}_{t}^{(k)}=\int_{W_{k}(M(E))} X_{t}(w, \cdot) 1_{\{\alpha(w) \geqslant-k\}} N^{J}(d w), \quad t \in \mathbf{R} .
$$

Очевидно, что $\left\{\bar{Y}_{t}^{(k)}: t \geqslant-k\right\}$ является процессом с иммиграцией, соответствуюшим SC-полугруппе $\left(N_{t}^{(k)}\right)_{t \geqslant 0}$, задаваемой соотношением $\int_{M(E)} e^{-\nu(f)} N_{t}^{(k)}(d \nu)=\exp \left\{-\int_{0}^{t} d s \int_{M(E)}\left(1-e^{-\nu\left(V_{s} f\right)}\right) 1_{\{\nu(E) \geqslant 1 / k\}} G(d \nu)\right\}$. 
Заметим, что для каждого $l>-k$ процесс $\left\{\bar{Y}_{t}^{(k)}:-k \leqslant t \leqslant l\right\}$ является почти наверное конечной суммой непрерывных справа траекторий и $\bar{Y}_{t}^{(k)} \rightarrow \bar{Y}_{t}^{J}$, монотонно возрастая, при $k \rightarrow \infty$, откуда, как и в работе [26], вытекает требуемый результат.

Теорема 6.3. Предположим, ито $\left(Q_{t}\right)_{t \geqslant 0}-$ переходная полугрупna $(\xi, \phi)$-суперпрочесса. Пусть $J \in \mathscr{E}\left(Q^{\circ}\right)$, u nycmb $\left\{Y_{t}^{J}: t \in \mathbf{R}\right\}$ onpedeляется соотношением (6.5). Для каждого $r>0$ положим

$$
Y_{t}^{r, J}=\int_{W(M(E))} w_{t} 1_{\{t \geqslant \alpha+r\}} N(d w), \quad t \in \mathbf{R} .
$$

Тогда $\left\{Y_{t}^{r, J}: t \in \mathbf{R}\right\}$ является почти наверное непрерывным справа стачионарным прочессом с иммиграчиеи $u Y_{t}^{r, J} \rightarrow Y_{t}^{J}$, монотонно возрастая почти наверное при $r \downarrow 0$ для каждого $t \in \mathbf{R}$.

Д оказ а тельст в о. Очевидно, что $J Q_{r}^{\circ} \in \mathscr{E}\left(Q^{\circ}\right)$ и $J Q_{r}^{\circ}=$ $J_{i}+J_{p} Q_{r}^{\circ}=J_{i}+\int_{0}^{\infty} H_{r} Q_{s}^{\circ} d s$. Используя (4.1), можно проверить, что мера Кузнецова на $W(M(E))$, определяемая соотношением $J Q_{r}^{\circ} \in \mathscr{E}\left(Q^{\circ}\right)$, является образом $\mathbf{Q}^{J}$ при отображении $\left\{w_{t}: t \in \mathbf{R}\right\} \mapsto\left\{w_{t} 1_{\{t>\alpha+r\}}: t \in \mathbf{R}\right\}$. Следовательно, $\left\{Y_{t}^{r, J}: t \in \mathbf{R}\right\}$ - стационарный процесс с иммиграцией, соответствующий $J Q_{r}^{\circ}$. По теореме 6.2 процесс $\left\{Y_{t}^{r, J}: t \in \mathbf{R}\right\}$ почти наверное непрерывен справа. Второе утверждение очевидно.

\section{СПИСОК ЛИТЕРАТУРЫ}

1. Athreya K.B., Ney P.E. Branching Processes. New York: Springer-Verlag, 1972, $287 \mathrm{p}$.

2. Cohn D. L. Measure Theory. Boston: Birkhäuser, 1980, 373 p.

3. Dawson D. A. Infinitely divisible random measures and superprocesses. - Progr. Probab., 1992, v. 31, p. 1-129.

4. Dawson D. A. Measure-valued Markov processes. - Lecture Notes in Math., 1993, v. 1541, p. $1-260$.

5. Dawson D. A., Ivanoff G. Branching diffusions and random measures. - Branching Processes. Ed. by A. Joffe and P. Ney. New York: Dekker, 1978, p. 61-103. (Adv. Probab. Relat. Topics, v. 5.)

6. Dellacherie C., Maisonneuve B., Meyer P. A. Probabilités et Potentiel. Ch. 17-24. Paris: Hermann, 1992.

7. Dynkin E. B. Three classes of infinite dimensional diffusion processes. - J. Funct. Anal., 1989 , v. 86 , p. $75-110$.

8. Dynkin E. B. Branching particle systems and superprocesses. - Ann. Probab., 1991, v. 19 , p. $1157-1194$.

9. Evans S. The entrance space of a Markov branching process conditioned on nonextinction. - Canad. Math. Bull., 1992, v. 35, p. 70-74.

10. Evans S. Two representations of conditioned superprocess. - Proc. Roy. Soc. Edinburgh. Sect. A, 1993, v. 123, p. 959-971.

11. Fitzsimmons $P$. J. Construction and regularity of measure-valued Markov branching processes. - Israel J. Math., 1998, v. 64, p. 337-361.

12. Fitzsimmons $P$.J. On the martingale problem for measure-valued Markov branching processes. - Seminar on Stochastic Processes, 1991. Ed. by E. Cinlar et al. Boston: Birkhäuser, 1992, p. 39-51. 
13. Fitzsimmons P. J., Maisonneuve B. Excessive measures and Markov processes with random birth and death. - Probab. Theory Relat. Fields, 1986, v. 72, p. 319-336.

14. Getoor R. K. Excessive Measures. Boston: Birkhäuser, 1990, 189 p.

15. Getoor R.K., Glover J. Constructing Markov processes with random times of birth and death. - Seminar on Stochastic Processes, 1986. Ed. by E. Cinlar et al. Boston: Birkhäuser, 1987, p. 35-69.

16. Gorostiza L. G., Lopez-Mimbela J. A. The multitype measure branching process. Adv. Appl. Probab., 1990, v. 22, p. 49-67.

17. Gorostiza L.G., Roelly S. Some properties of the multitype measure branching process. - Stochastic Process. Appl., 1990, v. 37, № 2, p. 259-274.

18. Hong W. M., Li Z. H. A central limit theorem for super Brownian motion with super Brownian immigration. - J. Appl. Probab., 1999, v. 36, p. 1218-1224.

19. Iscoe I. A weighted occupation time for a class of measure-valued branching processes. - Probab. Theory Relat. Fields, 1986, v. 71, p. 85-116.

20. Kallenberg $O$. Random Measures. Berlin: Akademie-Verlag, 1975, 104 p.

21. Kawazu K., Watanabe $S$. Branching processes with immigration and related limit theorems. - Теория вероятн. и ее примен., 1971, т. 16, в. 1, с. 34-51.

22. Кузнецов C.E. Построение марковских процессов со случайными моментами рождения и гибели. - Теория вероятн. и ее примен., 1973, т. 18, в. 3, с. 596-601.

23. Li $Z$. $H$. A note on the multitype measure branching process. - Adv. Appl. Probab., 1992 , v. 24 , p. $496-498$.

24. $L i$ Z. H. Measure-valued branching processes with immigration. - Stochastic Process. Appl., 1992, v. 43, p. 249-264.

25. $L i \quad Z$. H. Convolution semigroups associated with measure-valued branching processes. - Chinese Sci. Bull., (English Ed.), 1996, v. 41, p. 276-280.

26. Li Z. H. Immigration structures associated with Dawson-Watanabe superprocesses. - Stochastic Process. Appl., 1996, v. 62, p. 73-86.

27. $L i Z$. H. Immigration processes associated with branching particle systems. - Adv. Appl. Probab., 1998, v. 30, p. 657-675.

28. $L i Z$. $H$. Entrance laws for Dawson-Watanabe superprocesses with nonlocal branching. - Acta Math. Sci., (English Ed.), 1998, v. 18, p. 449-456.

29. Li Z. H., Shiga T. Measure-valued branching diffusions: immigrations, excursions and limit theorems. - J. Math. Kyoto Univ., 1995, v. 35, p. 233-274.

30. Perkins E.A. Conditional Dawson-Watanabe processes and Fleming-Viot processes. - Seminar on Stochastic Processes, 1991. Ed. by E. Cinlar et al., Boston: Birkhäuser, 1992, p. 143-156.

31. Sharpe M.J. General Theory of Markov Processes. Boston: Academic Press, 1988, $419 \mathrm{p}$.

32. Shiga T. A stochastic equation based on a Poisson system for a class of measurevalued diffusion processes. - J. Math. Kyoto Univ., 1990, v. 30, p. 245-279.

33. Shiga T., Watanabe $S$. Bessel diffusions as a one-parameter family of diffusion processes. - Z. Wahrscheinlichkeitstheor. verw. Geb., 1973, B. 27, S. 37-46.

34. Silverstein $M$. L. Continuous state branching semigroups. - Z. Wahrscheinlichkeitstheor. verw. Geb., 1969, B. 9, S. 235-257.

35. Watanabe $S$. A limit theorem of branching processes and continuous state branching processes. - J. Math. Kyoto Univ., 1968, v. 8, p. 141-167.

Поступила в редакцию 24.III.1999 